\title{
Opposing transcriptomic gradients explain orthogonal maps in human visual areas
}

\author{
Zonglei Zhen¹,2, Jesse Gomez ${ }^{\star 3,4}$, Kevin S. Weiner ${ }^{3,4}$ \\ 1. Beijing Key Laboratory of Applied Experimental Psychology, Beijing Normal University, Beijing, China \\ 2. Faculty of Psychology, Beijing Normal University, Beijing, China \\ 3. Helen Wills Neuroscience Institute, University of California Berkeley, Berkeley, California, United States of \\ America \\ 4. Psychology Department, University of California Berkeley, Berkeley, California, United States of America \\ * These authors contributed equally
}

\begin{abstract}
Opposing transcriptomic gradients explain the large-scale organization of cortex. Here, we show that opposing transcriptomic gradients also explain the finescale organization of orthogonal maps in human visual areas. We propose a model relating transcriptomics, cell density, and function, which predicts that specific cortical locations within these visual maps are microanatomically distinct and differentially susceptible to genetic mutations. We conclude with histological and translational data that support both predictions.
\end{abstract}

\section{Maint Text}

Active transcription of a small set of genes contributes to the large-scale arealization of functional hierarchies and gradients in human cortex. Two recent studies reveal that both the broad layout of functional regions ${ }^{1}$ and even the hierarchical ordering of regions within a processing stream ${ }^{2}$ are accurately described by genetic transcription that produces opposed, linear gradients in cortex. However, whether this broad relationship extends to smaller spatial scales is unknown. Thus, the goal of the present study was to answer the following fundamental question: What role do genes play in the organization of functional gradients, or topographic maps, within a single human cortical area?

While genetic gradients (e.g., Ephrins) during gestation establish the topography of connectivity in visual cortex ${ }^{3}$, the role that additional genes play in the layout of topographic maps within cortical areas in the adult brain is less well understood. To fill this gap in knowledge, we employed a dataset ${ }^{4}$ detailing the transcriptomic landscape of adult human cortex from the Allen Human Brain Atlas (AHBA). Early visual areas (V1, V2, V3) were used as target areas because they contain two, well-characterized orthogonal maps of eccentricity and polar angle 5 (Fig 1a) that can be modeled in the absence of functional data 6 . Using tools from our previous work ${ }^{2}$, tissue samples and functional areas were aligned to the same cortical surface.

We tested the a priori hypothesis that opposed linear gradients are not only a feature employed by cortex at the large-scale ${ }^{1,2}$, but also at finer spatial scales within 
cortical areas. We identified tissue samples $(n=136)$ from the AHBA located within human early visual areas that form linear gradients correlated with eccentricity or polar angle. To minimize false alarms, we selected only the top $1 \%$ of positively and negatively correlated genes ranked by the negative log of their $p$-values as in previous work ${ }^{2}$. Example genes whose expression correlated positively (higher expression in periphery) or negatively (lower expression in periphery) with increasing eccentricity are illustrated in Fig 1b. In each area, we observe significant correlations (r's > 0.6 , p's < 0.001 ) between the magnitude of genetic transcription and either polar angle or eccentricity, suggesting that the orthogonal maps of receptive field (RF) properties are described by equally orthogonal genetic gradients, which is consistent with our hypothesis. All correlations were between 29.5 and 40.2 standard deviations above their respective bootstrapped $(n=1000)$ null distributions (gray line in Fig 1c-d; Online Methods).

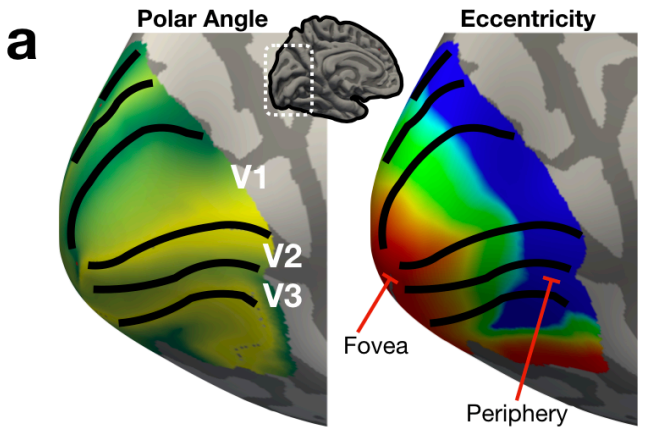

C
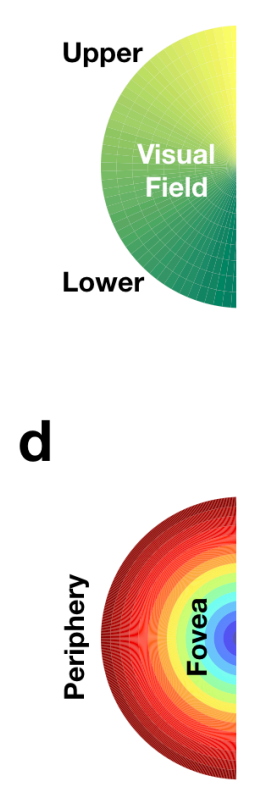

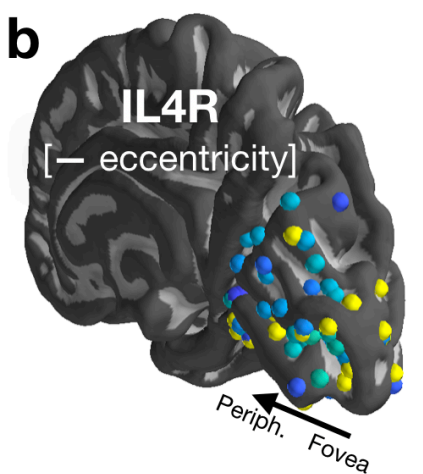

V2

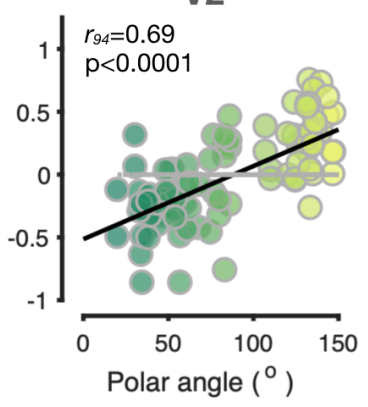

V2

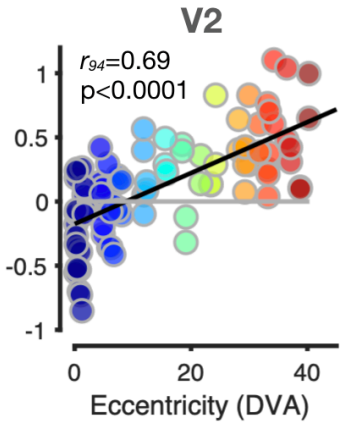

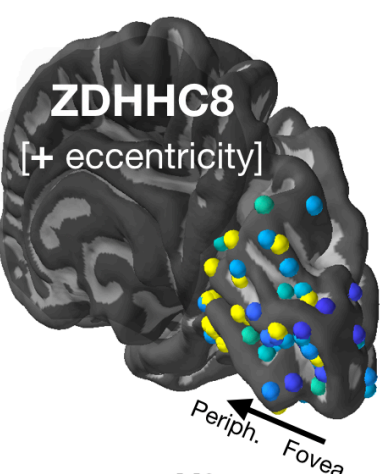

V3

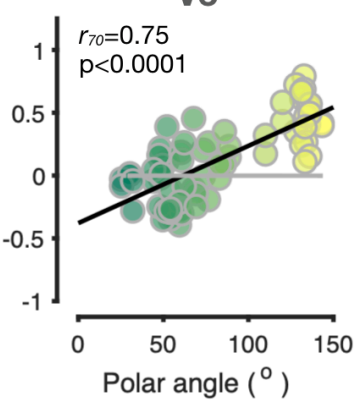

V3

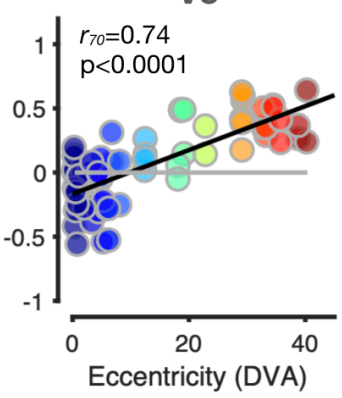

Figure 1: Opposing transcriptomic gradients explain orthogonal maps in human visual areas. (a) Example orthogonal maps of polar angle and eccentricity on an inflated cortical surface. Areas V1, V2, 
and V3 are defined (Online Methods). (b) Right hemisphere tissue samples of the AHBA projected onto the cortical surface. Inset bar displays colormap of normalized expression magnitude. Example genes whose transcription levels form either a negative gradient (left) or positive gradient (right) in relation to eccentricity. (c) Correlations between a tissue sample's polar angle preference and the mean expression of the top $1 \%$ of genes. For illustration purposes, the positive and negative gradients have been combined by inverting the sign of the negative gradient. As illustrated in Fig S1, the same result occurs when calculating correlations separately for each gradient. Black line: Line of best fit. Gray line: Average correlation from a bootstrapping $(\mathrm{N}=1000)$ procedure in which genes were randomly selected (Online Methods). (d) Same as (c) but for the underlying eccentricity value of each tissue sample. DVA: degrees of visual angle.

Interestingly, the identified genes are largely area- and map-specific. When calculating the dice coefficient between the genes contributing to the layout of eccentricity and polar angle within each area, there is little ( 3\%) overlap (dice of V1: 0.12; V2: 0.07; V3: 0.07; see Fig S2 for overlap between areas). The identified genes (Table S1) are independent of those identified previously that contribute to the largescale arealization of the visual processing hierarchy (Fig S2), indicating that cortical organization employs linear transcription gradients at multiple spatial scales using unique sets of genes. Additionally, polar angle and eccentricity measurements of one area (e.g., V1) do not predict $(-0.33<$ r's < 0.34, p's > 0.05; Figs S3-S6) the gene expression in another area (e.g., V2 or V3).
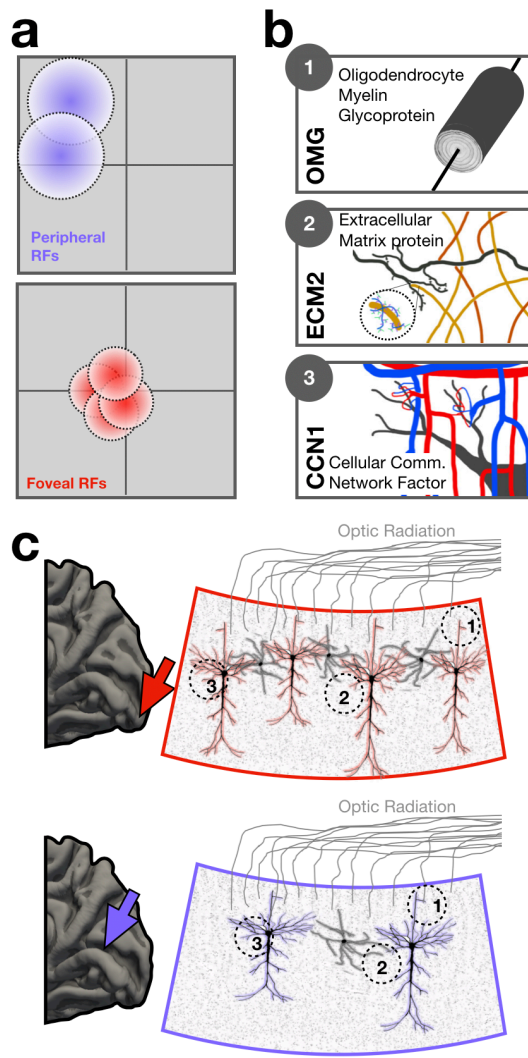

b
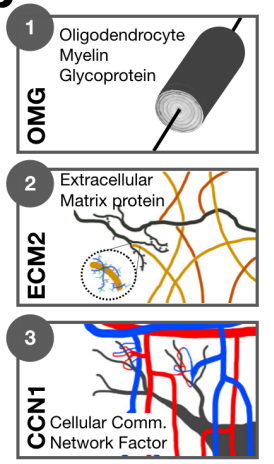

Optic Radiation

2.1
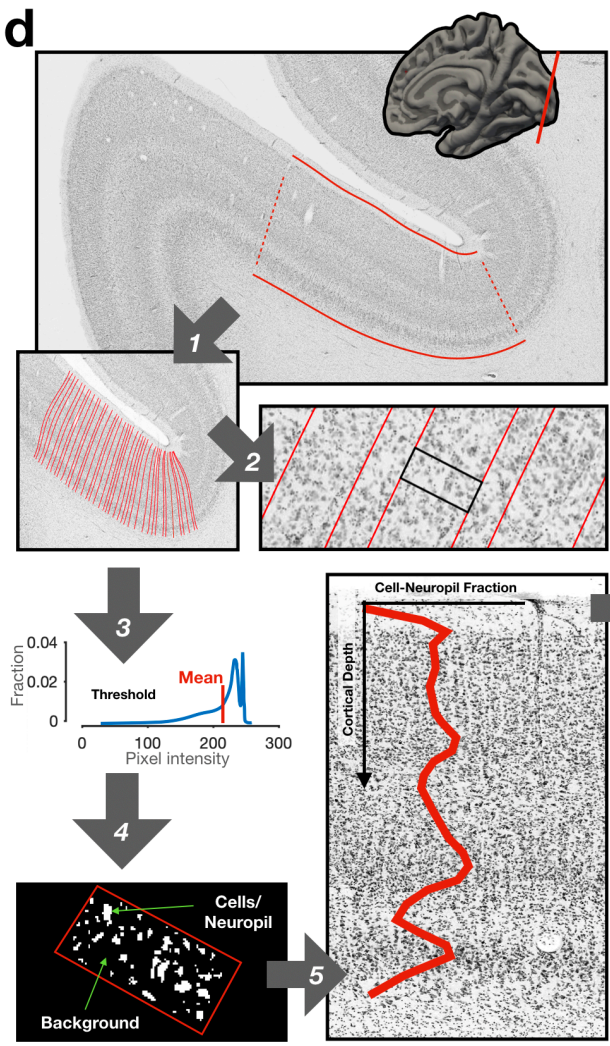

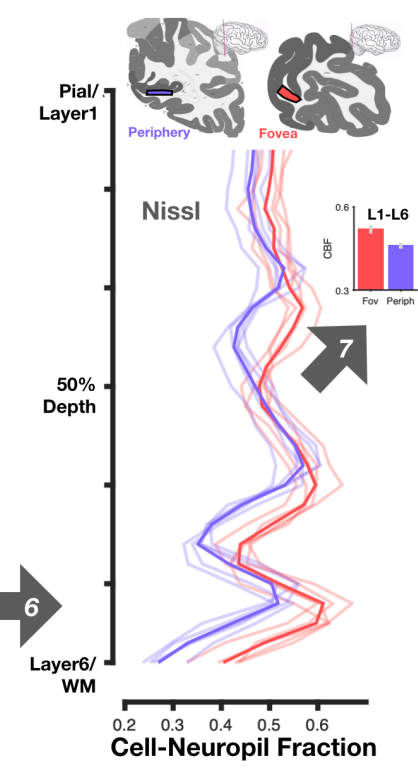

e

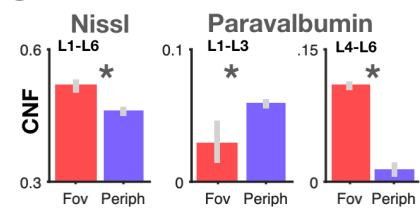

Figure 2: Validating tissue differences between foveal and peripheral cortex predicted by a functional-genetic model. (a) Schematic showing that receptive fields (RFs) are denser and smaller in 
foveal (red) compared to peripheral (purple) cortex. (b) Identified genes with increased expression in foveal compared to peripheral cortex (Table S1) suggests that higher RF density in foveal cortex is associated with higher expression of genes that regulate macromolecular tissue structures such as myelin (OMG), extracellular matrix material (ECM2; collagen, yellow curves; glycoproteins (blue/purple inset), and capillary junctions (CCN1). (c) A functional-genetic model resulting from (a) and (b) hypothesizes that higher RF density and increased expression of genes controlling key tissue structures will result in higher cellular/neuropil density in foveal cortex (posterior calcarine sulcus, red arrow) compared to peripheral (anterior calcarine sulcus, purple arrow). Right: Schematic illustration of cellular/ neuropil density differences between foveal (top) and peripheral (bottom) cortex. Colors: neurons. Gray: interneurons. Dotted circles (1-3): Where proteins of the genes in (b) are likely localized. (d) Basic steps of the approach developed here to quantify cytoarchitecture from coronal Nissl stains, which derives the fraction of pixels above background which we deem cell-neuropil fraction (CNF) along cortical traversals modeled to follow the organization of cortical columns. See Online Methods for description of the pipeline resulting in average CNF measurements across cortical layers (upper right inset) and histological slices. Red: foveal; Purple: peripheral slices of pericalcarine cortex. Dark contours represent the mean (step 6). Upper insets indicate the location of the histological slices from which each ROI (colored rectangles) was defined. (e) Left: Bar graph indicates that foveal portions have a significantly higher $(p<$. 005) CNF (0.52 +/- 0.02) compared to peripheral portions $(0.45+/-0.02)$ from Nissl stained sections. Right: Control analyses of paravalbumin stains show that CNF varied by cortical layer (Layers 1-3, peripheral $>$ foveal, $t(8)=2.72, p<0.03$; Layers $4-6$, foveal $>$ peripheral, $t(8)=5.2, p<0.001)$ indicating that foveal and peripheral cortical slices do not always vary by a global mean difference simply as a result of potential processing biases of the histological tissue (Online Methods).

The latter finding is particularly surprising considering that the representation of eccentricity is shared across areas V1-V3. We hypothesized that the identified genes may have different roles in the development of features that contribute to functional differences between areas and within a given topographic map. Two parsimonious features fit these criteria. Firstly, RFs are smaller and denser in foveal compared to peripheral cortex, as well as smaller in V1 compared to V2 and V3 (Fig 2a) ${ }^{7}$. Secondly, negative eccentricity gradient genes code for many microstructural proteins (Fig 2b) whose expression also decreases from fovea to periphery. Together, denser RFs and microstructure-promoting transcription generate a functional-genetic model (Fig 2c) that makes the explicit prediction that human foveal cortex has an increased density of cells and relevant neuropil to support the increased fidelity of vision associated with foveal processing. Employing a new histological quantification approach (Fig 2d), we successfully demonstrate differences in cell/neuropil density between foveal and peripheral V1 (Fig 2d, 2e) for the first time in humans, which is consistent with measurements from non-human primates ${ }^{8}$.

This functional-genetic model also makes the translational prediction that mutations in a subset of the identified genes should produce specific visual field deficits, likely unevenly across a given map. We verify the feasibility of this prediction with CXorf58 (Fig 3), an X chromosome gene whose expression negatively correlated with eccentricity and has been linked to retinitis pigmentosa (RP), which results in perceptual deficits of the peripheral visual field. Mutations in CXorf58 may also explain recent findings ${ }^{9}$ showing that females with Turner Syndrome (TS) - a condition in which an $\mathrm{X}$ chromosome is damaged or missing ${ }^{10}$ - present with deficits in RF coverage of the peripheral visual field specifically in V2 and V3, where CXorf58 is 
negatively correlated with eccentricity (Fig 3). Additional mutations in SLITRK4 and RAl2 - both $X$ chromosome genes involved in axonogenesis ${ }^{11}$ and cell growth ${ }^{12}$ and identified here - could adversely impact peripheral representations where tissue microstructure reductions would hinder the fidelity of neural circuits underlying the pooling of visual information.

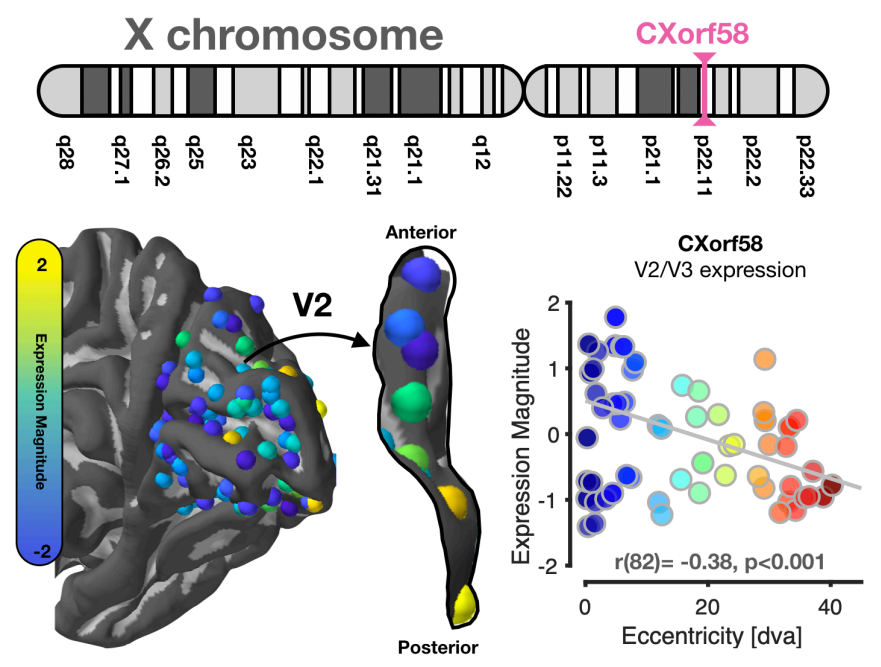

Figure 3: Translational insights into the role of genetic mutations in the loss of peripheral vision. The functional-genetic model makes the translational prediction that mutations in a subset of the identified genes should produce specific visual field deficits. Top: The location of CXorf58 on the short arm of the $X$ chromosome. Mutations in CXorf58 have been linked to retinitis pigmentosa (RP), which results in peripheral vision deficits. Bottom left: Expression pattern of CXorf58 within the human occipital lobe. Expression within dorsal V2 is emphasized given recent findings showing that patients with Turner syndrome have reduced peripheral visual field coverage in V2 and V3 compared to controls. Bottom right: Expression pattern of CXorf58 negatively correlates with eccentricity. We propose that CXorf58 mutations may not only be linked to deficits associated with RP, but also Turner syndrome.

Altogether, our results suggest a new rule of cortical organization in which the adult brain employs opposed transcriptional gradients at multiple spatial scales: across the cortex ${ }^{1}$, across areas within processing hierarchies ${ }^{2}$, and now across maps within a single area. Previously, we hypothesized that large-scale transcription gradients contributed to the differences in population RFs across areas of the visual processing hierarchy. Here, we improve the resolution of this hypothesis by an order of magnitude to the anatomical scale of cellular structures and the functional scale of RFs within a single visual map. While transcriptomic gradients contribute to the layout of RF properties within and across field maps, additional evidence suggests that such transcription shows protracted development ${ }^{2}$, and that RFs are even sculpted through experience and viewing behaviors ${ }^{13}$. Thus, experience likely modulates the transcription of genes involved in structural mechanisms underlying RFs. Future work will add additional features such as connectivity ${ }^{3}$ and cortical layering 17 to the present model, which will continue to deepen our understanding of the balance between transcriptomic and experiential contributions to the structure and function of human cortex. 


\section{Online Methods}

Data

All analyzed data were curated from the following open datasets:

1.Allen Human Brain Atlas (AHBA) Microarray Data: http://human.brain-map.org

2.Neuropythy atlas of human visual cortex: https://github.com/noahbenson/neuropythy

3.BrainSpan histological stains: http://www.brainspan.org/static/atlas

Defining eccentricity and polar angle maps in areas V1, V2, and V3

Human visual areas are defined by two orthogonal functional maps: one of a receptive field's (RF) polar angle describing its polar position along the circular image of the retina, and one of eccentricity, describing its distance from the center of the visual field (the fovea). While maps of eccentricity are shared across early visual areas, the transition of one area to another is based on differences in polar angle 5 . Polar angle transitions between early visual areas occur with such regularity relative to the folding of cortex that a model based on an individual's cortical anatomy is sufficient to automatically define areas V1 through V3 as precisely as manual definitions of these areas $^{6}$. We applied this model to a cortical surface of the MNI152 brain, which is an anatomical average of human cortex from the Montreal Neurological Institute made from the average of 152 brains. This cortical space was chosen given that tissue samples from the donor brains of the AHBA have been aligned to the same MNI volumetric space. A cortical surface was produced for this anatomical volume using FreeSurfer ${ }^{14}$.

\section{AHBA Tissue Samples}

The AHBA provides transcription data across human cortex, pooled from six donors and processed via Microarray. Microarray samples underwent strict normalization and data quality checks through AHBA as described here: http:// human.brain-map.org. For data organization, preprocessing was identical to our previous work2. Importantly, we follow the standardized processing pipeline proposed by Arnatkeviciute, Fulcher, and Fornito ${ }^{15}$. Briefly, the raw microarray expression data for each of the six donor brains included the expression level of 29,131 genes profiled via 58,692 microarray probes. We implemented five preprocessing steps. First, probes were excluded that did not have either a) a gene symbol or b) an Entrez ID. This resulted in 20,737 genes. Second, the expression profiles of all the probes targeting the same gene were averaged. Third, in order to remove variability in gene expression across donors, gene expression values of tissue samples were normalized by calculating z-scores separately for each donor. Fourth, because previous studies did not identify significant inter-hemispheric transcriptional differences, data from both hemispheres were combined. Finally, comparing a tissue sample's genetic expression to either the eccentricity or polar angle of its underlying cortex required mapping these tissue samples to a common cortical space. Each sample from the AHBA was 
associated with a 3D coordinate from its donor's MRI brain volume and its corresponding coordinate $(x, y, z)$ in the MNI152 space. Each tissue sample was mapped to the nearest cortical surface vertex with a $5 \mathrm{~mm}$ distance threshold and assigned to the visual field map in which that surface vertex was contained. This resulted in the following number of tissue samples aligned to each visual area: 54 in V1, 47 in V2, and 35 in V3.

\section{Comparing gene transcription and visual field map properties}

With tissue samples and maps of eccentricity and polar angle having been mapped to the same cortical surface, we extracted the underlying eccentricity or polar angle from each tissue sample by taking the average of the vertices that were within a one voxel radius of the vertex to which the tissue sample had been mapped. As our previous study identified that genes distinguishing regions along a cortical processing hierarchy are organized into opposed, linear gradients ${ }^{2}$, we first tested the a priori hypothesis that opposed gradients would also exist within a single brain region to explain the orthogonal maps of eccentricity and polar angle of visual field maps. To that end, for each gene, we calculated the Pearson correlation between its expression magnitude across tissue samples within an area versus the mean eccentricity or polar angle of each tissue sample. Genes were then ranked by the negative log of their resulting $\mathrm{p}$-values. As in the previous study ${ }^{2}$, the top $1 \%$ of positively correlated and the top $1 \%$ of negatively correlated genes were kept. The average expression value of those top genes are illustrated in Fig 1. This was done separately for each visual field map. For example, the mean expression of the top 1\% $(n=200)$ of positively correlated genes in V1 is averaged within each tissue sample and then plotted against its eccentricity or polar angle (pink circles of Fig S1). For purposes of visualization, we combined the two gradients together into a single gradient by flipping the sign of the negative gradient for Fig 1, but the opposed gradient with their original signs are shown in Fig S1. A list of the top positive and negative gradient genes for eccentricity and polar angle are listed in Table S1 for each visual field map. By averaging across the top genes, we aim to capture a general pattern that is shared across many genes, rather than focusing on a single gene which is likely more susceptible to a Type II error. As a control analysis, we sought to generate a null distribution for each correlation using a bootstrapping approach. Across $n=1000$ bootstraps, we repeated each correlation in each field map but randomly selected the same number of genes as the main correlation (from the total population of 20,737 genes). We plot the averaged correlation in Fig 1 as gray lines for each field map. We calculated, in units of standard deviation, the distance that the main correlations were from the bootstrapped null distribution. These distances were very large, ranging from 29.5 to 40.2 standard deviations, underscoring the significance of the observed correlations.

\section{Comparing inter-regional and intra-regional gene expression}

In a previous study2, we identified $n=200$ genes that formed opposed positive ("ascending") and negative ("descending") gradients along the visual processing hierarchy of occipito-temporal cortex. We hypothesized that given that these genes were identified as those that distinguish regions within a processing stream, they 
should be unique, or non-overlapping, with the genes identified in the present study that are correlated with functional topography within a visual area. From these 200 inter-regional genes, and the intra-regional genes identified in each field map (2 orthogonal maps * 2 gradients * 200 genes per gradient $=800$ genes per field map), we calculated the dice coefficient to describe the overlap of these two sets per field map. The dice coefficient (DC) is calculated as twice the number of overlapping genes normalized by the size of both lists. In this way, the highest DC possible for the dataset at hand is 0.5 . The resulting $\mathrm{DC}$ for this analysis for V1, V2, and V3 is shown in Fig S2a. Of these 1000 total genes going into the DC analysis, approximately 2 overlapped. We also repeated this analysis for the list of intra-regional genes identified in two visual field maps. The resulting three DCs from the possible combination of comparisons for the three visual field maps is shown in Fig S2a. Next, to explicitly validate that the interregional genes identified in the previous study were not correlated with eccentricity or polar angle, separately for each of the "ascending" and "descending" gene gradients, we correlated the mean expression of these genes in the current tissue samples with eccentricity and polar angle in V1, V2, and V3. All of the resulting 12 correlations illustrated in Fig S2b were indeed non-significant: $-0.2<r$ 's $<0.22$, p-values $>0.18$. Lastly, we wanted to explicitly test if the genes identified from one region were correlated with functional properties in another region (Figs S3-S6). To do this, for example, we took the genes correlated with eccentricity in V2 and V3, removed the shared genes with V1 (e.g., genes that were also correlated with eccentricity in V1 were excluded) and then we correlated the expression of these unique V2N3 genes in tissue samples of $\mathrm{V} 1$ with their underlying eccentricity. For all cases, we find no correlation (Bonferroni correction for multiple comparisons) with the exception of V2-unique genes being correlated with eccentricity in the tissue samples of V1.

\section{Quantifying histological slices of human cortex with BrainWalker}

In order to test the feasibility of the hypothesis that gene transcription differences between foveal and peripheral representations within a single visual field map might induce microanatomical differences in the underlying cortical sheet, we developed a method to quantify human cytoarchitecture that a) was repeatable across different histological samples, b) could capture anatomical variation across cortical layers, and c) was importantly, observer-independent. To achieve this goal, we developed an analysis pipeline called BrainWalker that quantifies the fraction of cell bodies and neuropil (cell-neuropil fraction, CNF) above background noise in a given histological stain. Importantly, this software slides or "walks" across cortical layers and columns, producing a vectorized description of the CNF for a desired piece of cortical ribbon. The software works as follows, and is illustrated in Fig $2 \mathrm{~d}$. First, the user selects a ribbon of cortex to be quantified, tracing the middle of Layer 1 of cortex, and the corresponding length of the gray-white matter boundary (Fig $2 d$, box 1). The two boundaries are then split into 200 equally spaced points. Points in Layer 1 and the white matter boundary are modeled as positive and negative electric charges, respectively, and then equipotential lines are drawn connecting each corresponding point on the two boundaries (Fig 2d, arrow 1). These equipotential traversals are meant to model the shape of cortical columns traversing the cortical sheet, importantly fanning at gyral crowns ${ }^{16}$. Each traversal is then split into 30 equally spaced points, 
and then a window slides between two neighboring traversals along these 30 bins (Fig $2 \mathrm{~d}$, arrow 2). The underlying image (e.g., a Nissl stain) is thresholded at the mean pixel value to identify the "peaks" or puncta associated with cell bodies and neuropil structures (Fig 2d, arrow 3). While this threshold was somewhat arbitrary, a threshold was necessary to exclude irrelevant image structures such as holes from veins, tears in the tissue, or air bubbles on the mounting slide. These irrelevant values, or "background", where the white slide mount becomes visible can be seen as the large peaks in the histograms of Fig S8. Fig S8 also shows that the chosen threshold does not impact the interpretation of the findings: histograms of all pixel values from the foveal and peripheral slices indicate that foveal slices (red) have greater tissue content at the entire range of lower pixel values corresponding to cortical tissue all the way up to the mean pixel intensity (solid black line) which marks the beginning of brighter pixels representing the slide background. Differences between foveal and peripheral slices at these higher pixel intensities result simply from the cropping of the images; foveal slices had more of the underlying slide visible as the posterior slices of the occipital pole were smaller than more anterior slices. After this thresholding, at every bin, the CNF (fraction of pixels belonging to cell bodies and neuropil versus background) is quantified (Fig 2d, arrow 4). The 30-bin vector for each traversal is then averaged across traversals, producing an average CNF contour detailing CNF from Layer 1 of cortex to white matter (Fig 2d, arrow 5). In Fig 2d, an example CNF contour is overlaid on a Nissl-stained section of human V1; one can appreciate how the peaks and valleys of the CNF contour highlight the different cell body densities across and between layers.

To generate CNF contours from human visual cortex, we used the freely available, high-resolution, Nissl-stained and Paravalbumin-stained coronal slices of human visual cortex from the Allen Institute's BrainSpan Atlas (http:// www.brainspan.org/static/atlas). In order to compare foveal and peripheral cortex, where genetic transcription is most differentiated in the present data and resulting anatomical differences are likely to be largest, we extracted slices $(n=5)$ from the posterior Calcarine sulcus where foveal representations are located, and slices from the anterior Calcarine sulcus where the peripheral visual field is represented. To ensure that differences in overall stain intensity would not bias results, and to mirror the normalization steps for the transcriptional data, each slice was normalized by the mean pixel value of all the neighboring white matter pixels. On a region of the cortical ribbon spanning 1.25 centimeters, we extracted the CNF of each slice from V1 and plotted the CNF of each slice in Fig 2 comparing fovea and periphery for Nissl-stained cortex. We repeated this analysis with paravalbumin-stained cortex in Fig S6 (Fig 2e). For Nissl stains, we computed a simple two-sample t-test comparing the CNF between foveal $\mathrm{V} 1$ and peripheral V1 averaged across cortical layers (Fig 2e). For the paravalbumin stains, CNF varied by cortical layer, and t-tests were run comparing Layers 1-3 and Layers 4-6 separately (Fig 2e). Paravalbumin was analyzed not only as an additional feature that could vary by eccentricity, but as a control to ensure that foveal and peripheral cortical slices do not always vary by a global mean difference simply as a result of potential processing biases from the AHBA. 


\section{Intra-regional genes on the $X$ chromosome}

Turner syndrome is a condition affecting females in which the individual is either missing an X-chromosome (monosomy $\mathrm{X}$ ) or one of the $\mathrm{X}$-chromosomes is damaged. While the condition is hallmarked by a number of physical atypicalities, genes on the short arm of the X chromosome (e.g., short stature homeobox gene, SHOX) have been linked to the stunted bone and limb growth that characterizes the small stature of those with Turner syndrome. Recent research has demonstrated that females with Turner syndrome present with visual deficits in the coverage of the periphery ${ }^{9}$. Retinotopic mapping of population receptive fields ( $\mathrm{pRF}$ ) in these individuals using functional MRI revealed that in $\mathrm{V} 2$ and $\mathrm{V} 3$, Turner females show reduced pRF coverage of the periphery compared to control females. With this finding in mind, we performed a directed search for genes in V2 and V3 that correlated with eccentricity located on the $X$ chromosome, with special attention for those on the short arm of the chromosome. Table S2 shows the complete list of genes from V2 and V3 that were correlated with eccentricity and were located on the $X$ chromosome. In Fig 3 , we highlight gene CXorf58 (Chromosome $X$ open reading frame 58), which is not only located on the $X$ chromosome short arm, but is also a gene whose mutations are known to result in Retinitis Pigmentosa, which is characterized by the loss of peripheral vision. Other notable genes were SLITRK4, directly involved in regulating neurite outgrowth and arborization, as well as RAl2, involved in cell growth. Both of these genes likely contribute to the CNF differences we observe in typical adult cortex, and their mutation likely has especially adverse impact on peripheral representations where cell density is already lower compared to foveal cortex. 


\section{Bibliography}

1 Burt, J. B. et al. Hierarchy of transcriptomic specialization across human cortex captured by structural neuroimaging topography. Nature Neuroscience, doi:10.1038/s41593-018-0195-0 (2018). 2 Gomez, J., Zhen, Z. \& Weiner, K. S. Human visual cortex is organized along two genetically opposed hierarchical gradients with unique developmental and evolutionary origins. PLoS Biol 17, e3000362, doi:10.1371/journal.pbio.3000362 (2019).

3 Barone, P. D., C.; Berland, M.; Kennedy, H. Role of directed growth and target selection in the formation of cortical pathways: prenatal development of the projection of area V2 to area V4 in the monkey. The Journal of Comparative Neurology 374, 1-20 (1996).

$4 \quad$ Hawrylycz, M. J. et al. An anatomically comprehensive atlas of the adult human brain transcriptome. Nature, doi:10.1038/nature11405 (2012).

5 Wandell, B. A. \& Winawer, J. Imaging retinotopic maps in the human brain. Vision Res 51, 718-737 (2011).

6 Benson, N. C. \& Winawer, J. Bayesian analysis of retinotopic maps. Elife 7, doi:10.7554/eLife. 40224 (2018).

7 Wandell, B. A. \& Winawer, J. Computational neuroimaging and population receptive fields.

Trends in cognitive sciences 19, 349-357, doi:10.1016/j.tics.2015.03.009 (2015).

8 Collins, C. E., Airey, D. C., Young, N. A., Leitch, D. B. \& Kaas, J. H. Neuron densities vary across and within cortical areas in primates. Proc Natl Acad Sci U S A 107, 15927-15932, doi:10.1073/pnas. 1010356107 (2010).

9 Green, T. et al. X-chromosome insufficiency alters receptive fields across the human early visual cortex. The Journal of Neuroscience, doi:10.1523/jneurosci.2745-18.2019 (2019).

10 Stochholm, K., Juul, S., Juel, K., Naeraa, R. W. \& Gravholt, C. H. Prevalence, incidence, diagnostic delay, and mortality in Turner syndrome. J Clin Endocrinol Metab 91, 3897-3902, doi:10.1210/ jc.2006-0558 (2006).

11 Gaudet, P., Livstone, M. S., Lewis, S. E. \& Thomas, P. D. Phylogenetic-based propagation of functional annotations within the Gene Ontology consortium. Brief Bioinform 12, 449-462, doi:10.1093/ bib/bbr042 (2011).

12 Accogli, A. et al. A novel Xp22.13 microdeletion in Nance-Horan syndrome. Birth Defects Res 109, 866-868, doi:10.1002/bdr2.1032 (2017).

13 Gomez, J., Natu, V., Jeska, B., Barnett, M. \& Grill-Spector, K. Development differentially sculpts receptive fields across early and high-level human visual cortex. Nature Communications 9, doi:10.1038/ s41467-018-03166-3 (2018).

14 Dale, A. M., Fischl, B. \& Sereno, M. I. Cortical surface-based analysis. I. Segmentation and surface reconstruction. Neuroimage 9, 179-194, doi:10.1006/nimg.1998.0395 (1999).

15 Arnatkeviciute, A., Fulcher, B. D. \& Fornito, A. A practical guide to linking brain-wide gene expression and neuroimaging data. Neuroimage 189, 353-367, doi:10.1016/j.neuroimage.2019.01.011 (2019).

16 Smart, I. H. M. \& McSherry, G. M. Gyrus formation in the cerebral cortex of the ferret. Description of the internal histological changes. Journal of Anatomy 147, 27-43 (1986).

17 Kaschube, M. W., F.; Puhlmann, M.; Rathjen, S.; Schmidt, K.-F.; Geisel, T.; Lowel, S. The pattern of ocular dominance columns in cat primary visual cortex: intra- and interindividual variability of column spacing and its dependence on genetic background. European Journal of Neuroscience 18, 3251-3266, doi:10.1046/j.1460-9568.2003.02979.x (2003).

18 Miller, J. A. et al. Transcriptional landscape of the prenatal human brain. Nature, doi:10.1038/ nature13185 (2014).

19 Schleicher, A. Z., K.; Wree, A. A quantitative approach to cytoarchitectonics: software and hardware aspects of a system for the evaluation and analysis of structural inhomogeneities in nervous tissue. Journal of Neuroscience Methods 18, 221-235 (1986).

20 Takahata, T., Shukla, R., Yamamori, T. \& Kaas, J. H. Differential expression patterns of striate cortex-enriched genes among Old World, New World, and prosimian primates. Cereb Cortex 22, 2313-2321, doi:10.1093/cercor/bhr308 (2012). 

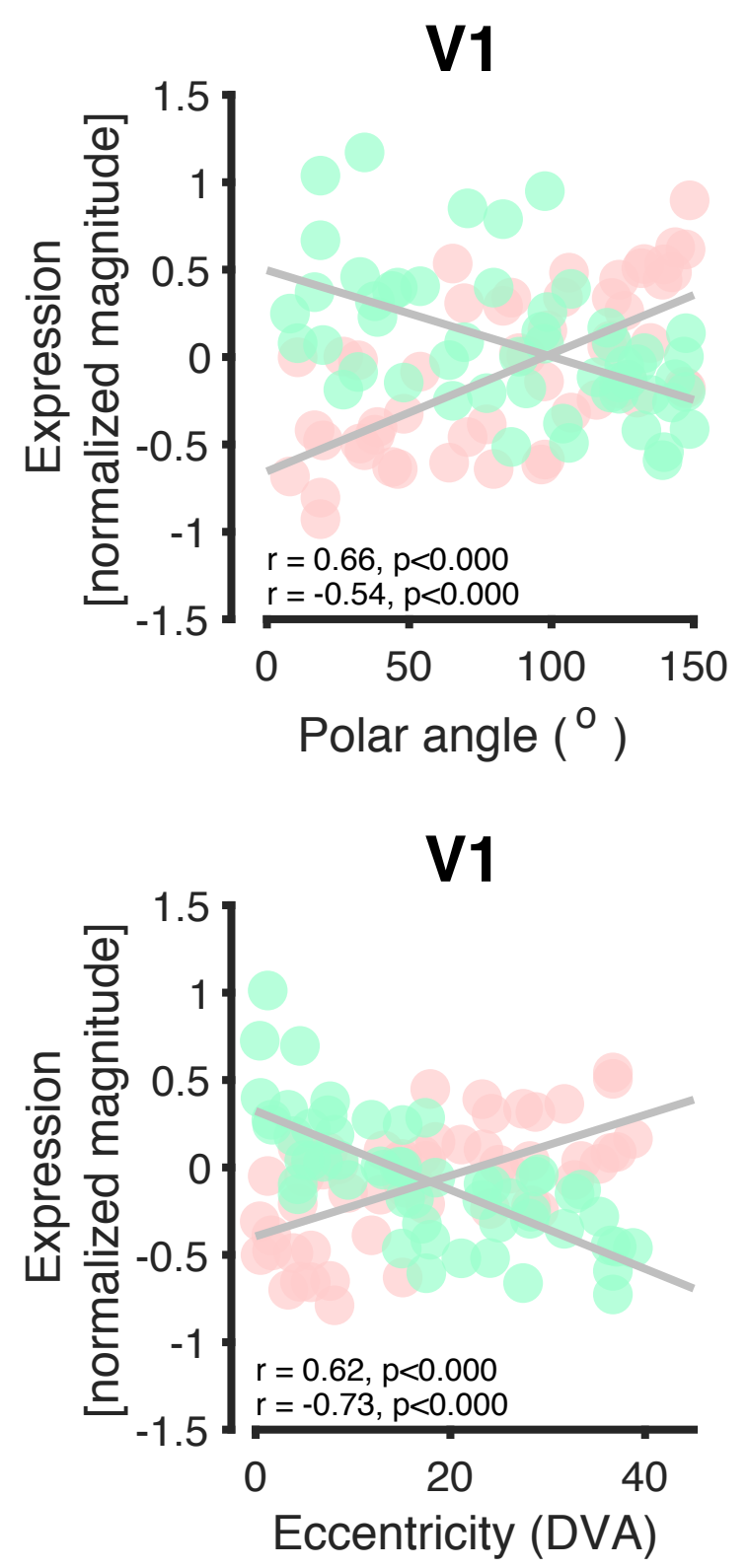
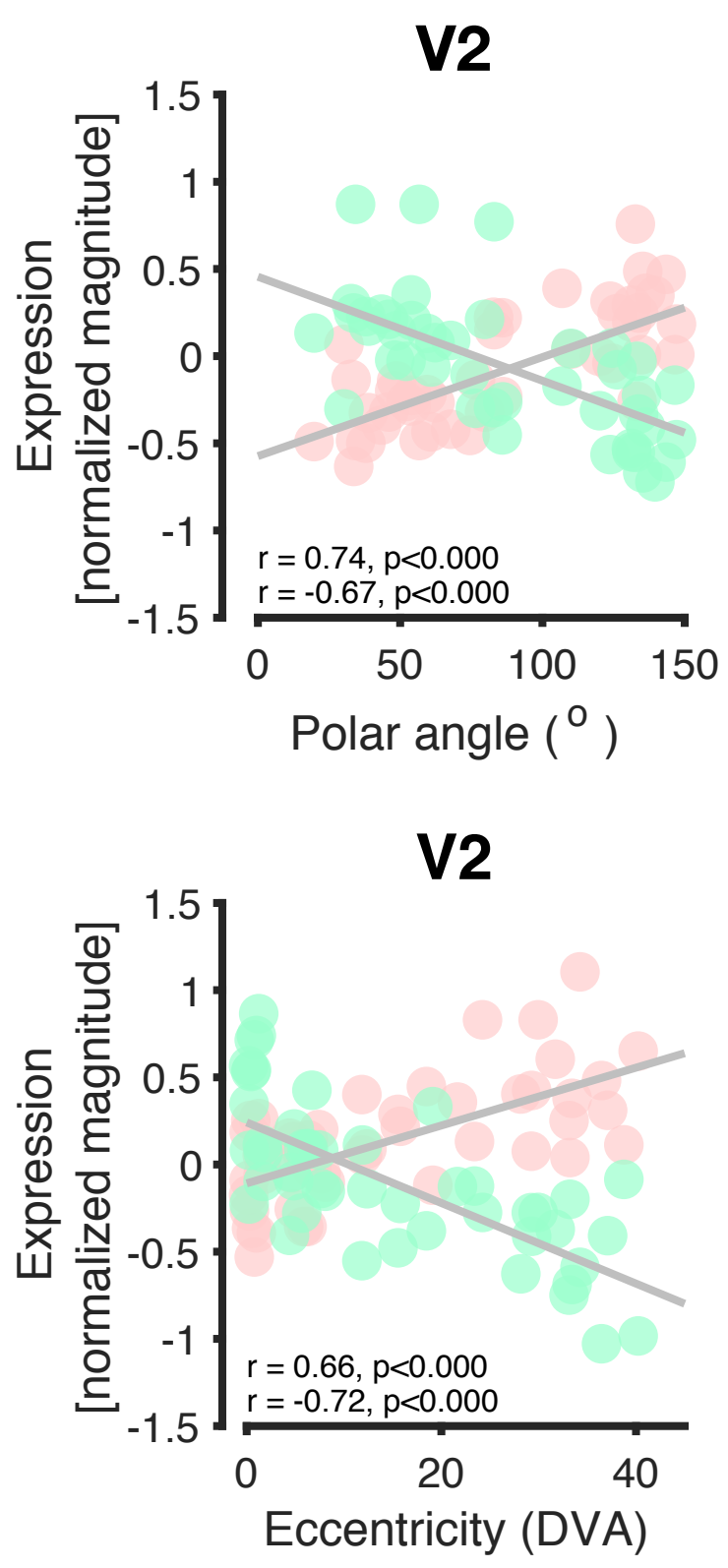

V3
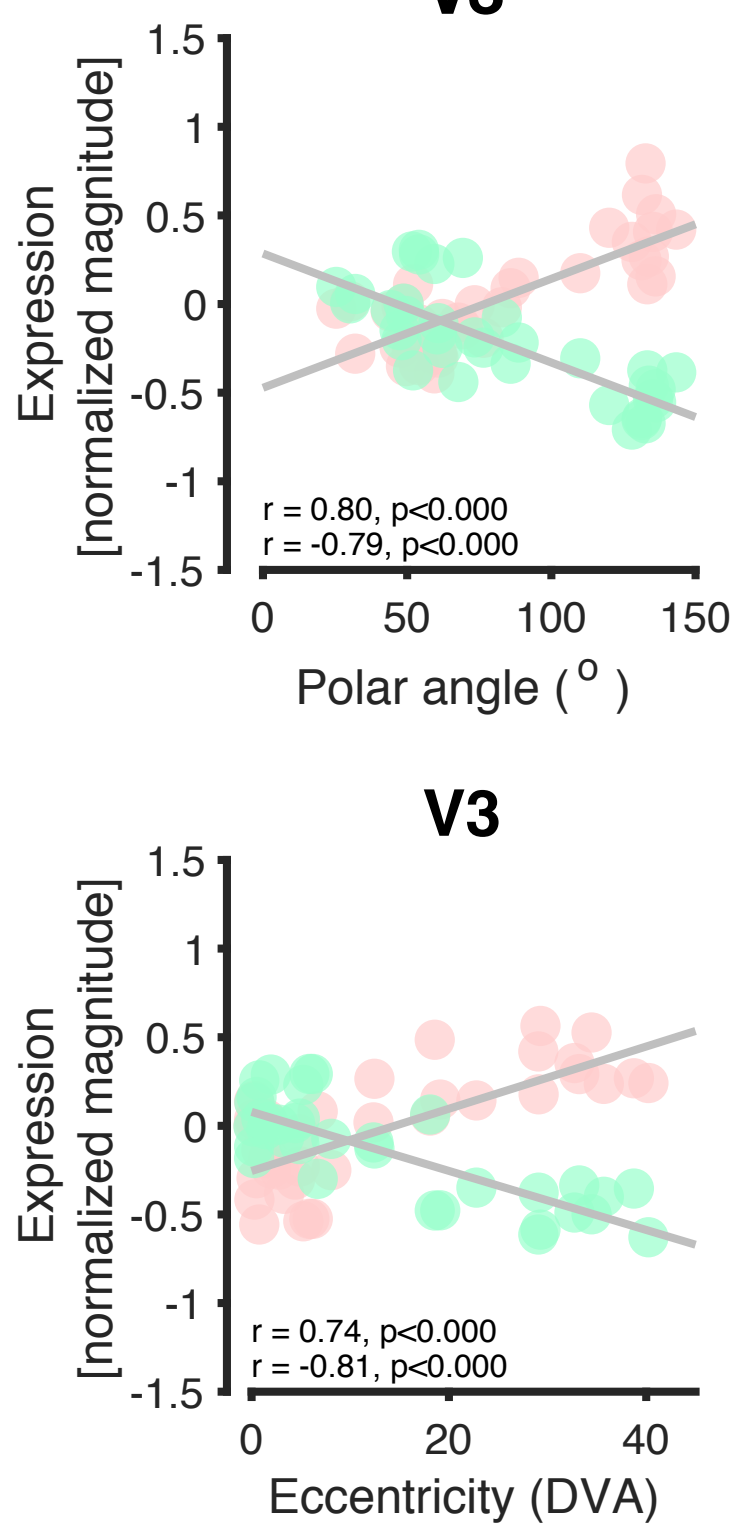

Supplementary Figure 1: Correlations between functional representation (polar angle and eccentricity) and the expression of positive and negative gradient genes. The mean expression of positive gradient genes within tissue samples are represented in pink dots, while the mean expression magnitude of negative gradient genes are shown in green. Pearson correlations and the resulting $p$-values are shown as inset text in each plot. Line of best fit for the positive and negative correlations are overlaid as gray lines. For each gradient, there are the following number of tissue samples: V1 ( $n=54)$, V2 ( $n=47)$, V3 $(n=35)$. (a) Polar Angle. (b) Eccentricity. 
a

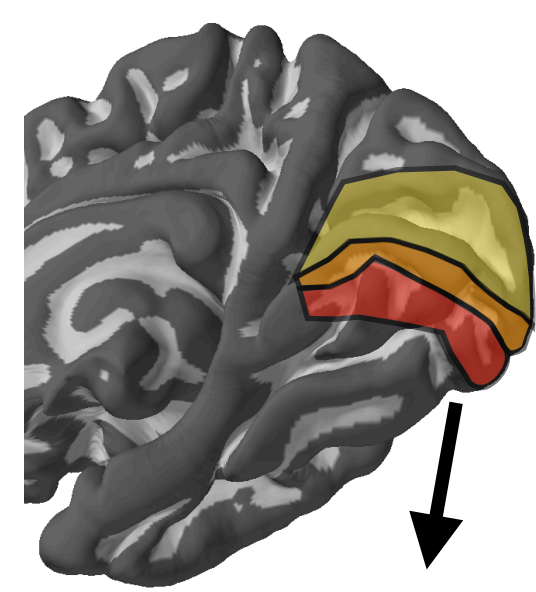

Inter-regional
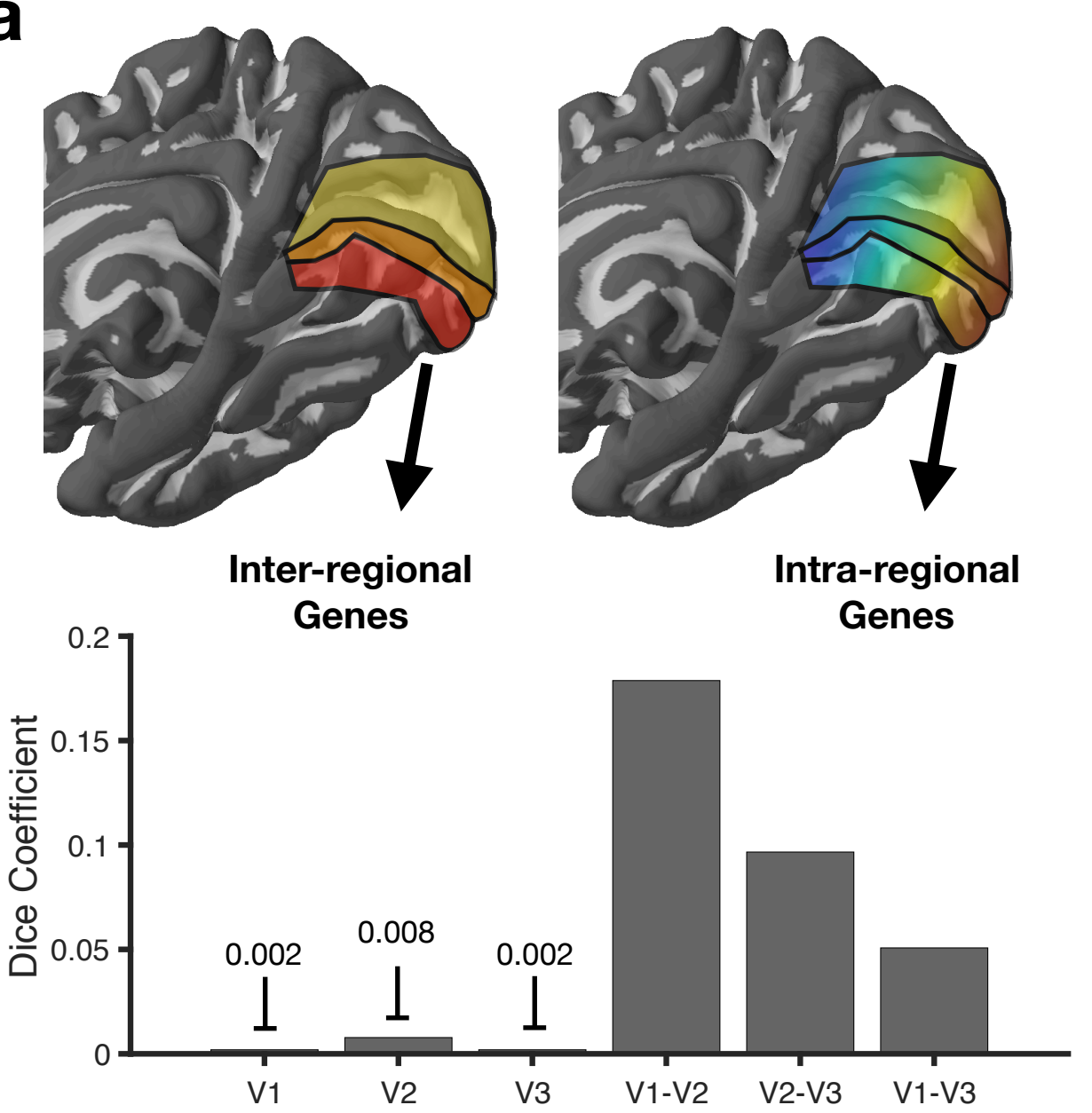

Genes
Intra-regional

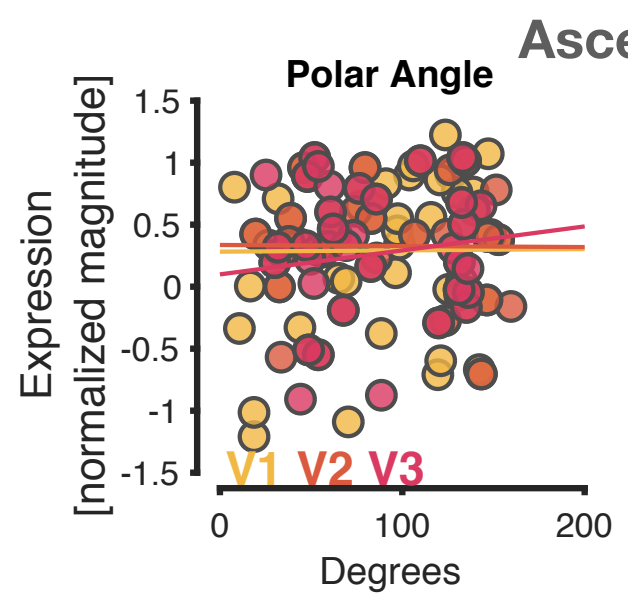

b

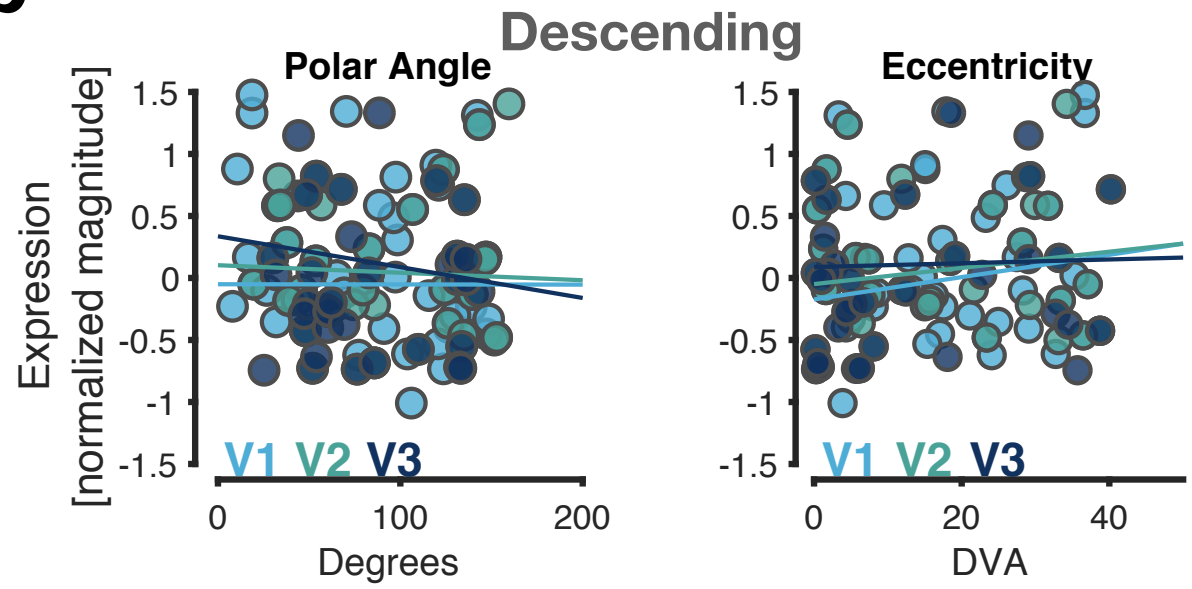

Degrees

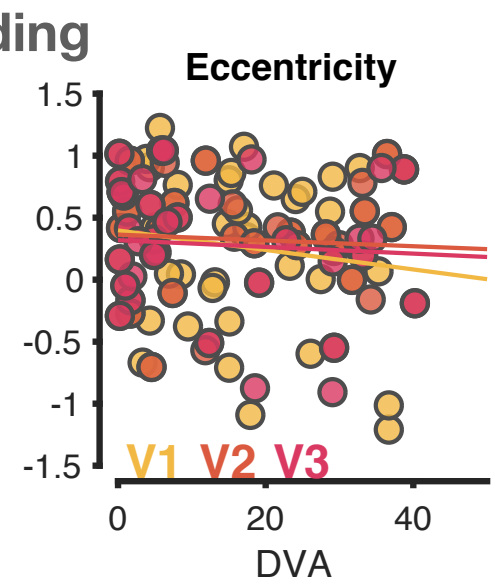

Supplementary Figure 2: Genes involved in hierarchical ordering of the ventral visual processing stream (inter-areal genes) are distinct from those correlated with eccentricity or polar angle (intra-areal genes). (a) Left: Dice coefficients summarizing the overlap of intra-areal genes in V1 through V3 with previously identified inter-regional genes that distinguish regions across the visual processing hierarchy. Right: The overlap of intraregional genes of one visual area relative to the others. V1 and V2 share the most genes. Overall, the intra-regional genes are largely restricted to a given area. (b) Mean gene expression levels relative to polar angle (left) or eccentricity (right) values for the previously identified inter-regional genes belonging to the descending (top) and ascending (bottom) gradients $(-0.2<$ r's $<0.22$, p-values $>0.18)$. That is, there were two clusters of genes identified in Gomez, Zhen, \& Weiner 2019 that varied linearly in expression level as one traversed the visual processing hierarchy (e.g., from V1 to ventral temporal cortex): one cluster that ascended in expression level (positive correlation) and one that descended (negative correlation) in expression level from early to late positions of the visual processing hierarchy. The expression of these inter-regional genes for either the descending (top) or ascending (bottom) gradient were not correlated with polar angle or eccentricity within areas V1, V2, or V3. 


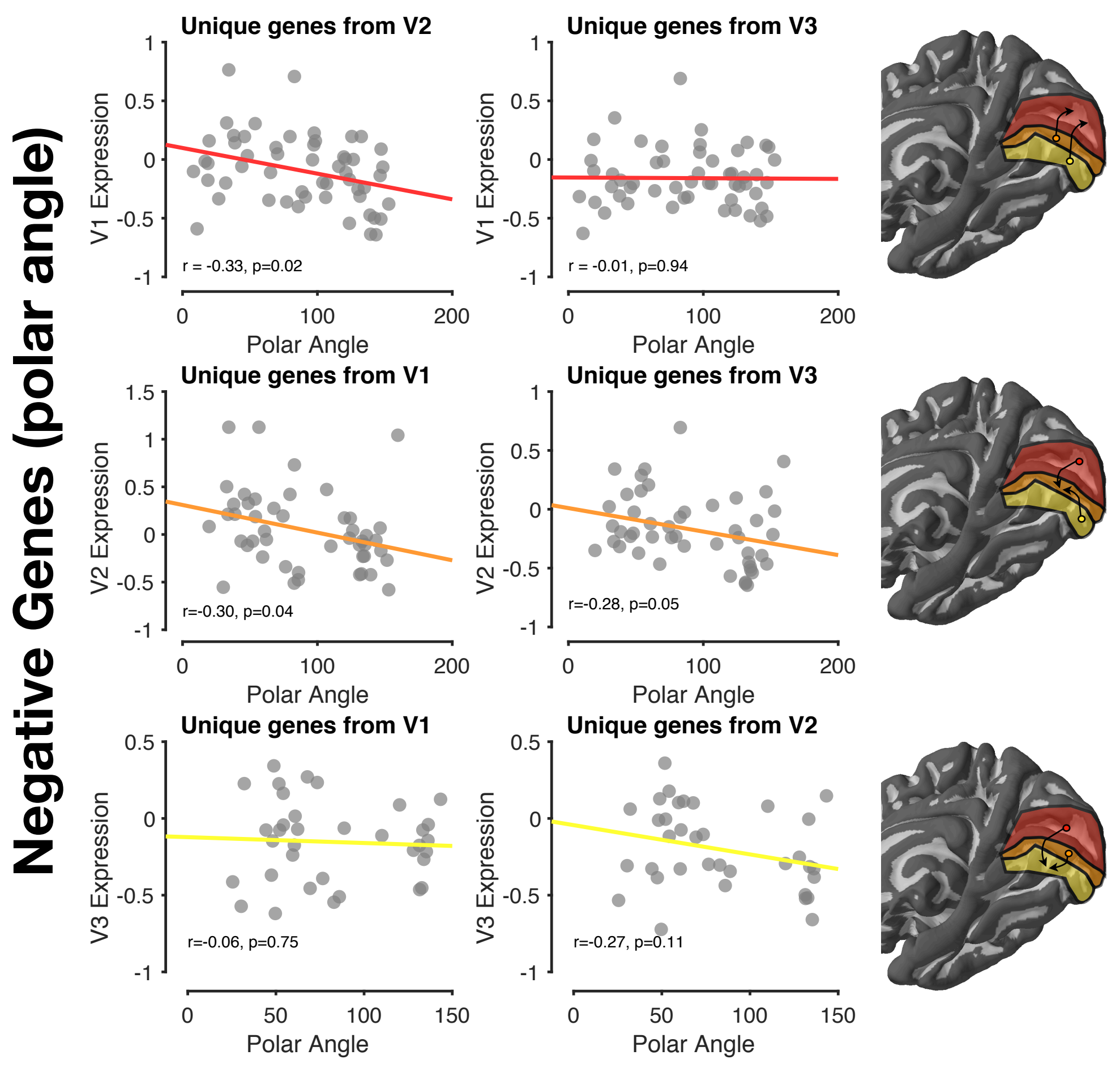

Supplementary Figure 3:

Correlations between polar

angle and expression of

negative genes from

neighboring field maps. Polar-

angle-correlated genes from each

field map, while slightly

overlapping, were largely a unique set of genes for each field map. To test whether the expression of genes from neighboring field maps were correlated with polar angle in the left out map, we took, for example, the genes from V2 and V3 correlated with polar angle, removed the overlapping genes that were correlated with polar angle in V1, and then correlated the mean expression of the remaining set of genes with polar angle in V1. The line of best fit is shown in each plot, and the resulting Pearson and $p$-values are inset as black text. Each gray circle is a tissue sample within a given field map. 

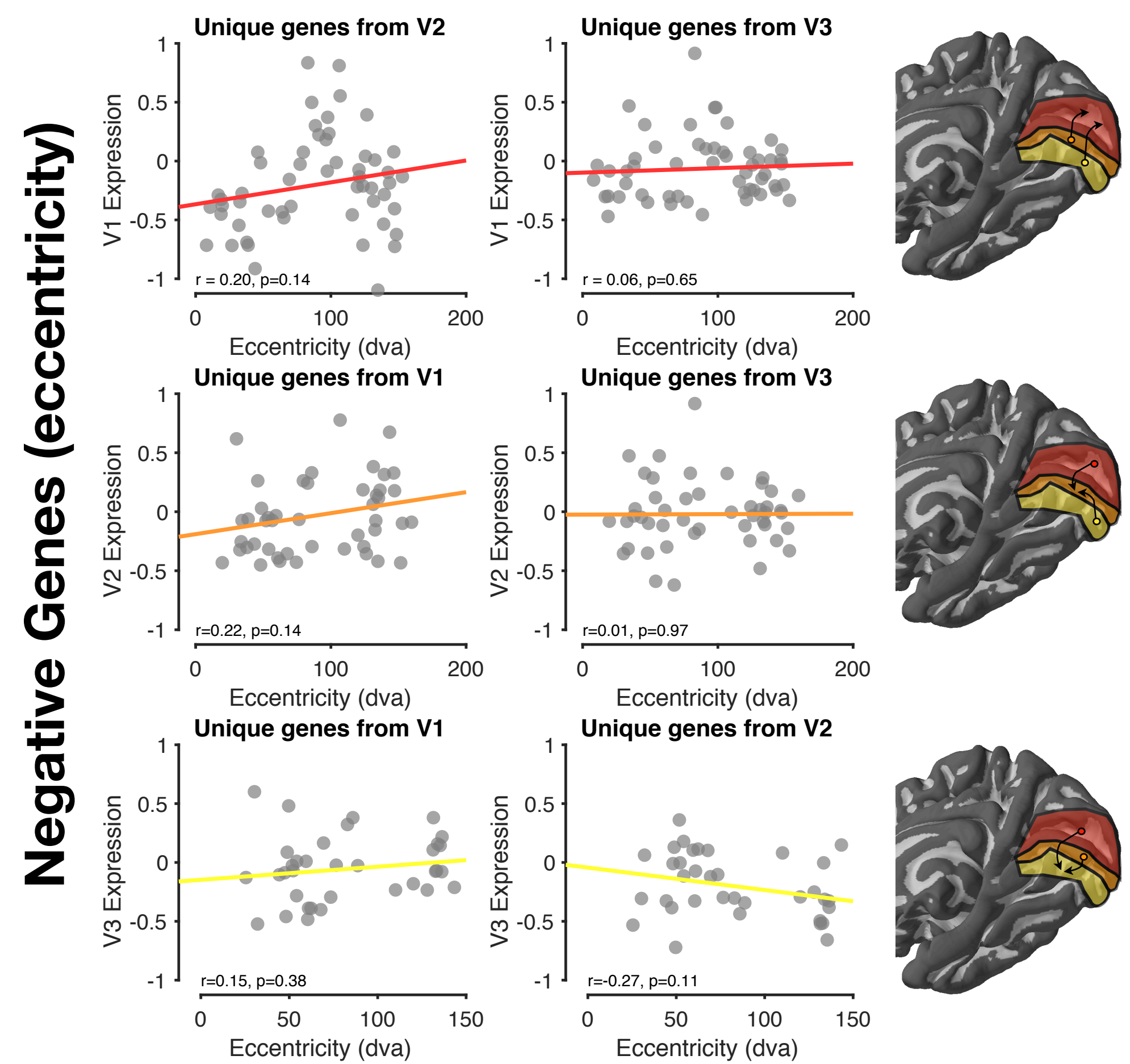

Supplementary Figure 4:

Correlations between

eccentricity and expression of

negative genes from

neighboring field maps.

Eccentricity-correlated genes from each field map, while slightly

overlapping, were largely a unique set of genes for each field map. To test whether the expression of genes from neighboring field maps were correlated with eccentricity in the left out map, we took, for example, the genes from V2 and V3 correlated with eccentricity, removed the overlapping genes that were correlated with eccentricity in V1, and then correlated the mean expression of the remaining set of genes with eccentricity in V1. The line of best fit is shown in each plot, and the resulting Pearson and $p$-values are inset as black text. Each gray circle is a tissue sample within a given field map. 

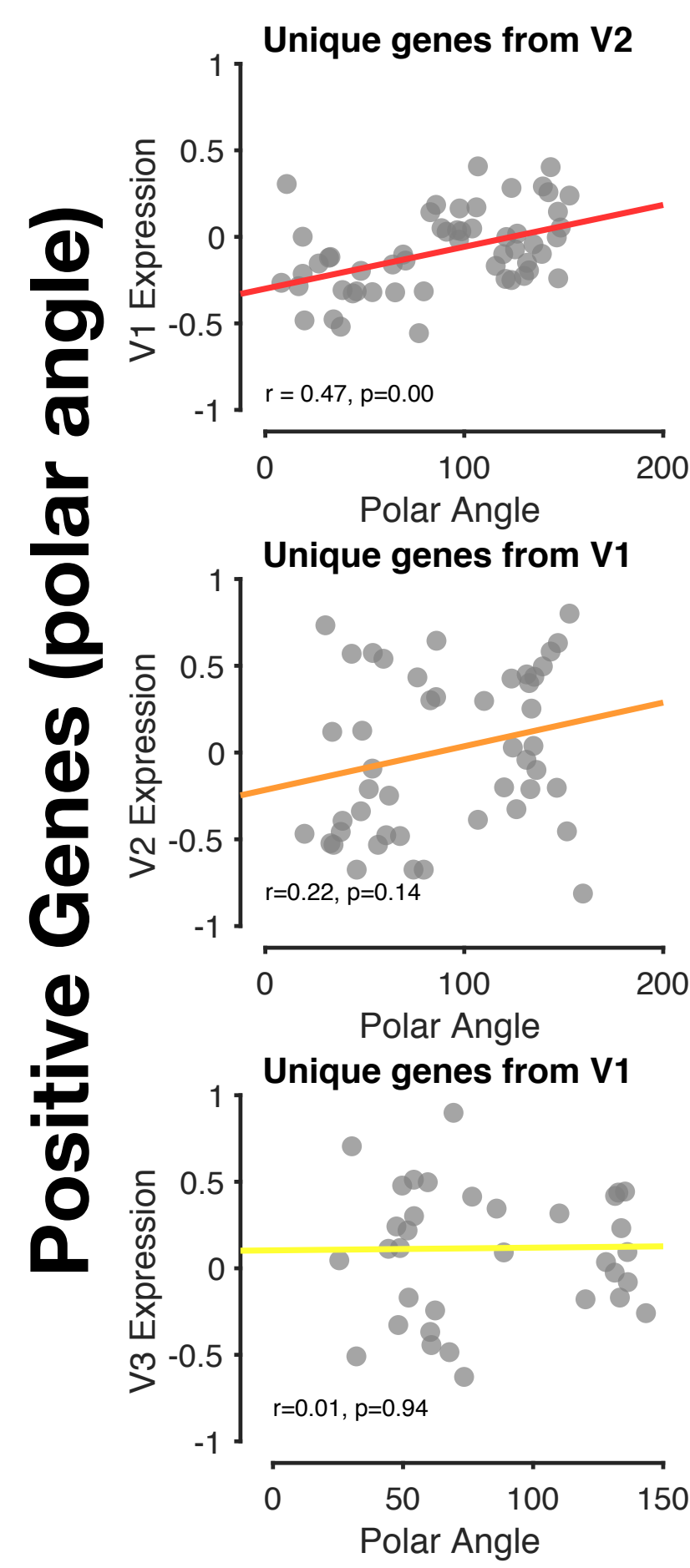
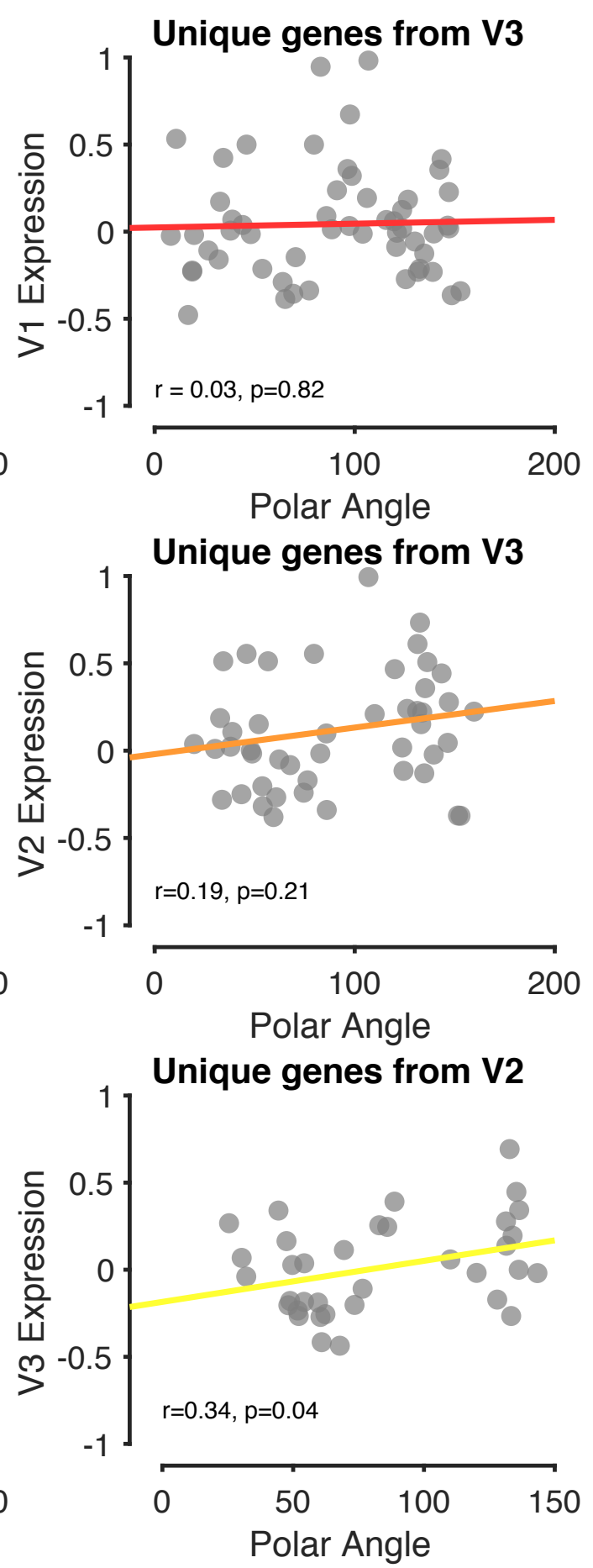
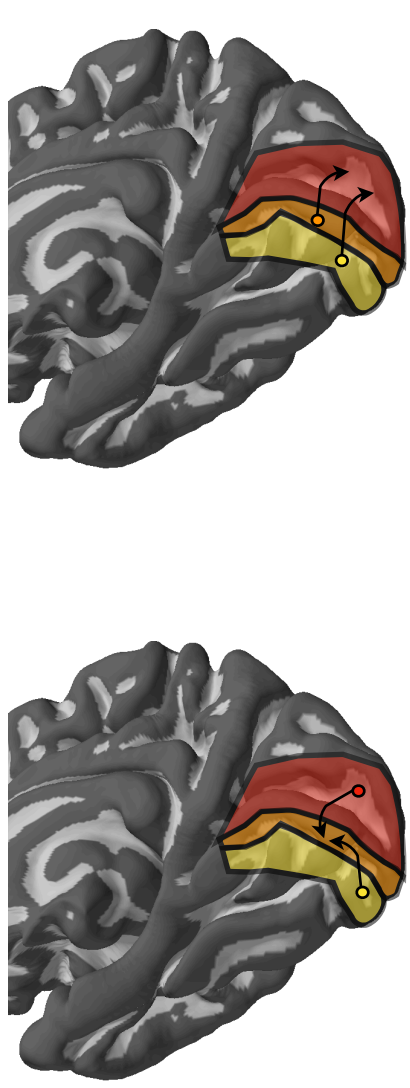

Supplementary Figure 5: Correlations between polar angle and expression of positive genes from neighboring field maps. Polar-angle-correlated genes from each field map, while slightly overlapping, were largely a unique set of genes for each field map. To test whether the expression of genes from neighboring field maps were correlated with polar angle in the left out map, we took, for example, the genes from V2 and V3 that were correlated with polar angle, removed the overlapping genes that were correlated with polar angle in V1, and then correlated the mean expression of the remaining set of genes with polar angle in V1. The line of best fit is shown in each plot, and the resulting Pearson and p-values are inset as black text. Each gray circle is a tissue sample within a given field map.

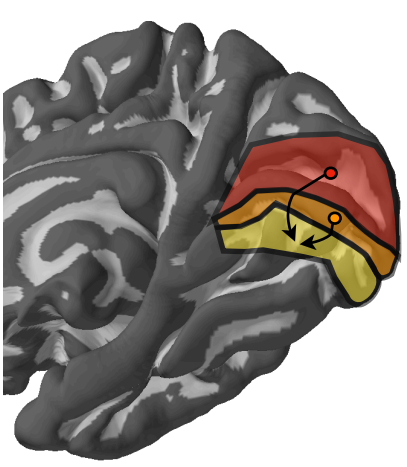




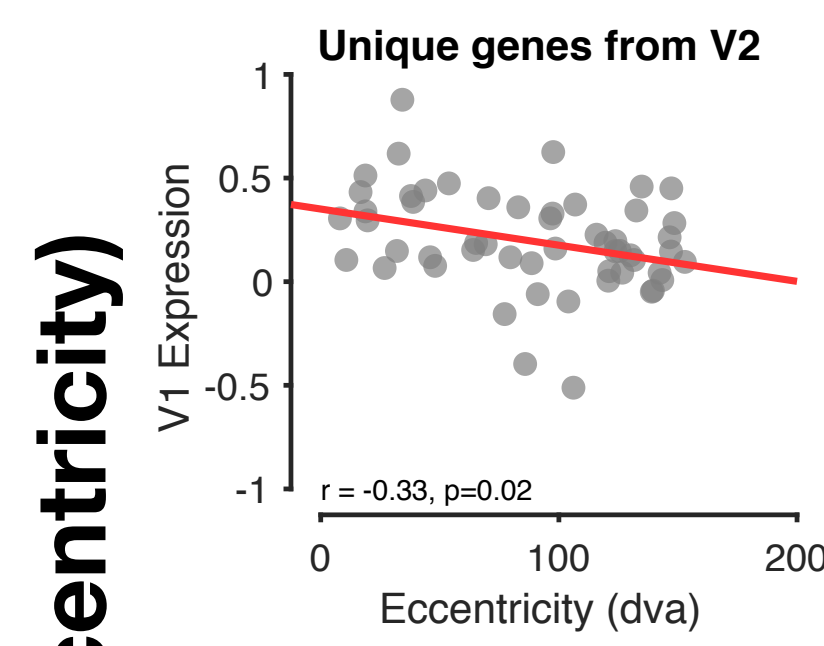

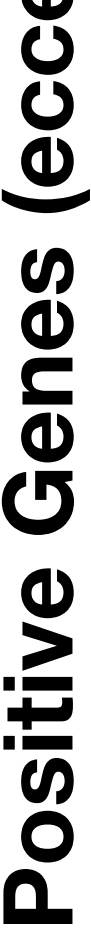
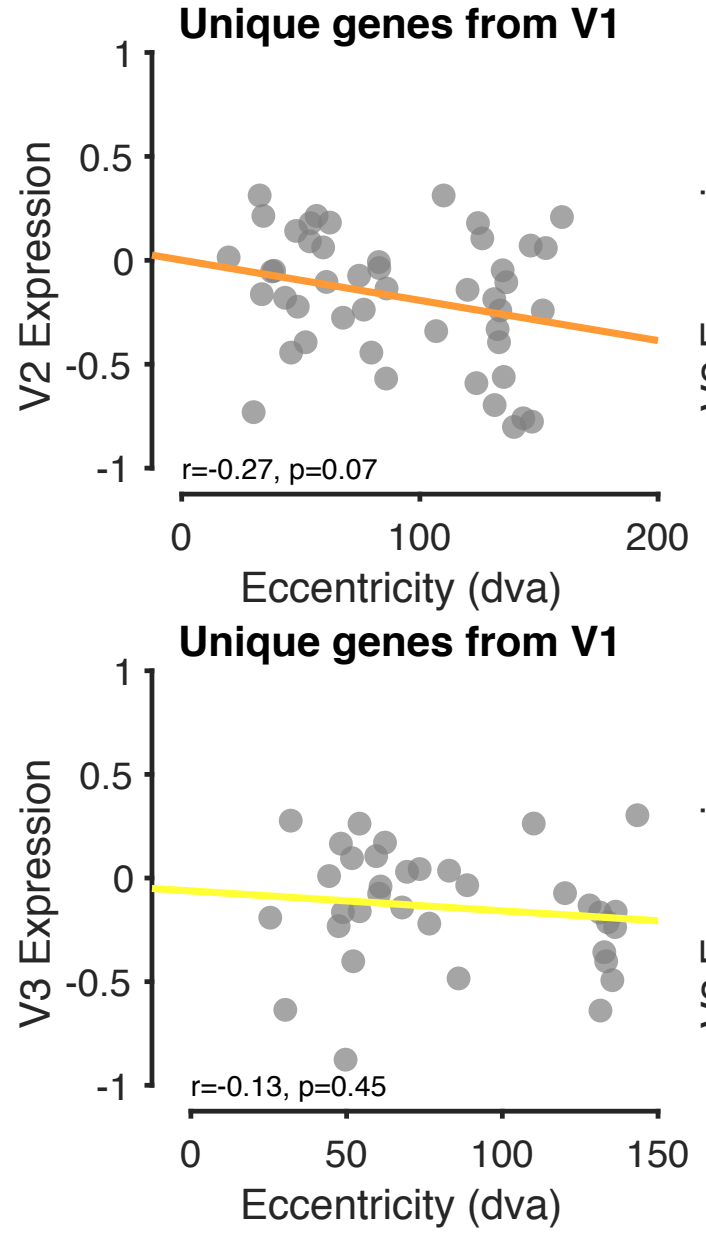
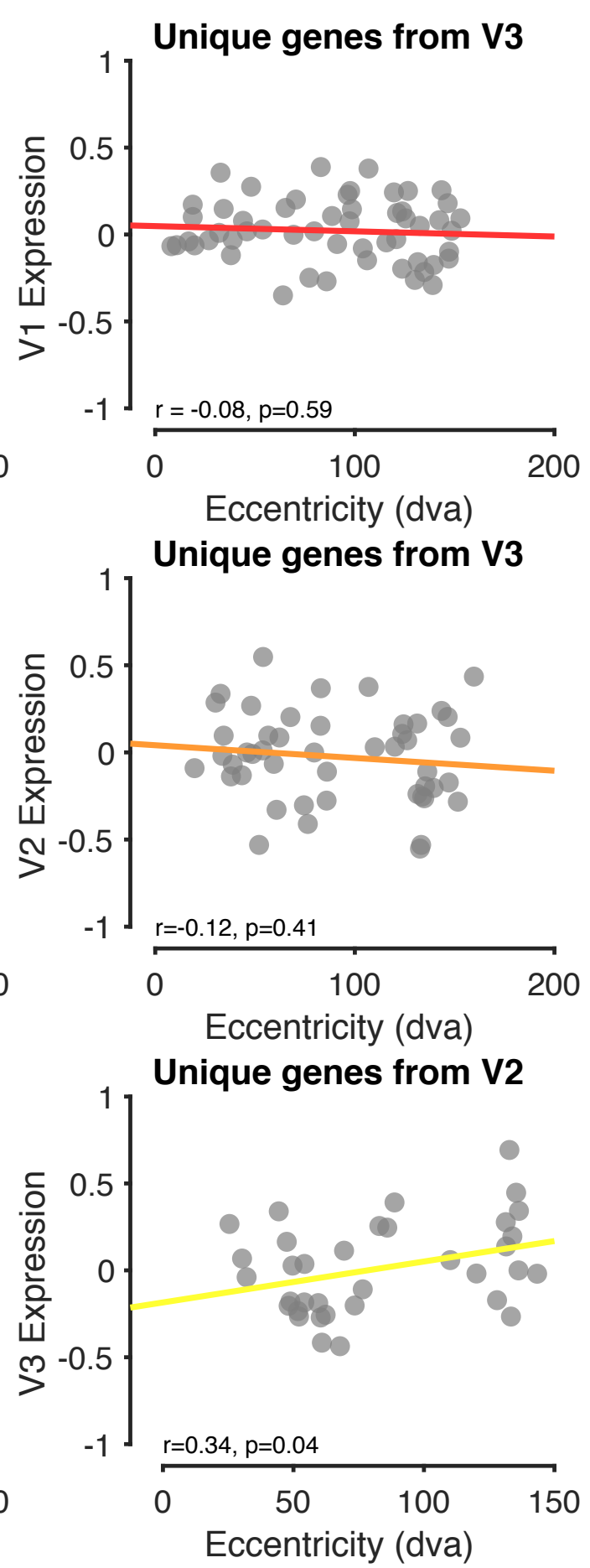
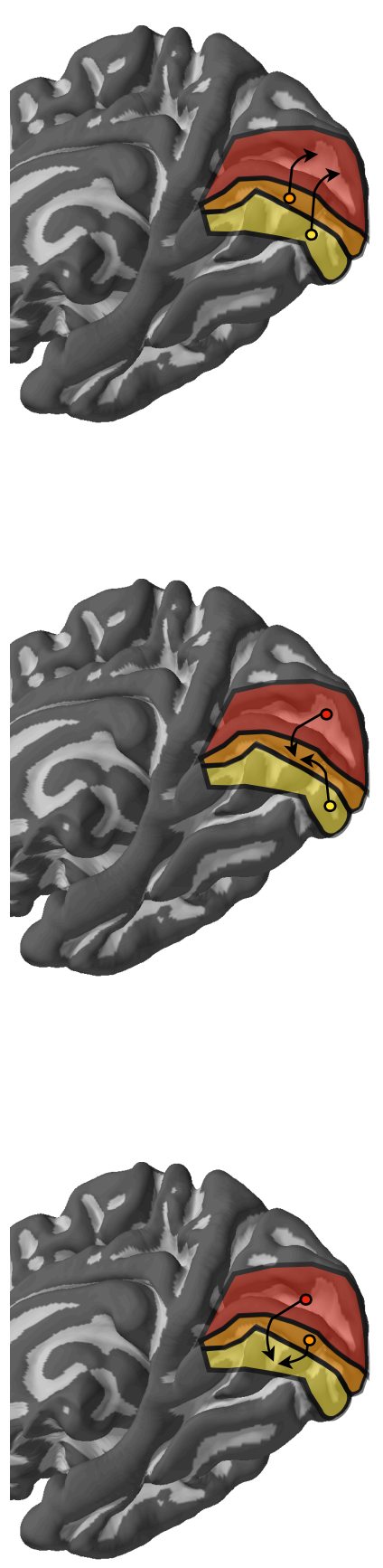

Supplementary Figure 6:

Correlations between

eccentricity and expression of positive genes from neighboring

field maps. Eccentricity-correlated

genes from each field map, while slightly overlapping, were largely a unique set of genes for each field map. To test whether the expression of genes from neighboring field maps were correlated with eccentricity in the left out map, we took, for example, the genes from V2 and V3 that were correlated with eccentricity, removed the overlapping genes that were correlated with eccentricity in V1, and then correlated the mean expression of the remaining set of genes with eccentricity in $\mathrm{V} 1$. The line of best fit is shown in each plot, and the resulting Pearson and $p$-values are inset as black text. Each gray circle is a tissue sample within a given field map. 
Pial/

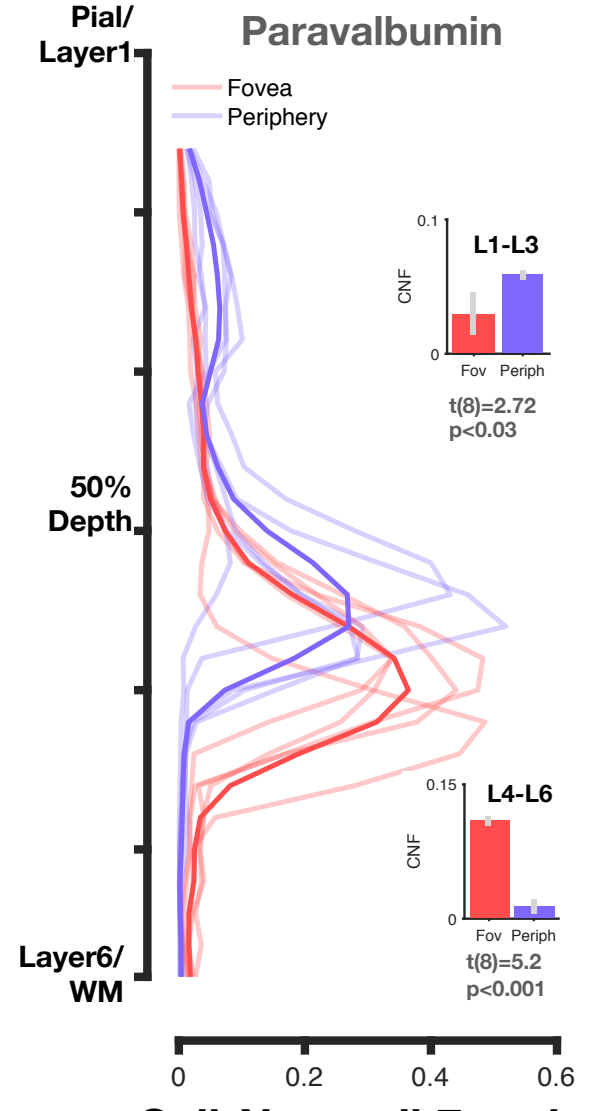

Cell-Neuropil Fraction

Supplementary Figure 7: Differential presence of paravalbumin between foveal and peripheral visual cortex. CNF contours stained for Paravalbumin

interneurons. We find that paravalbumin varies significantly between foveal and peripheral $V_{1}$, with lower CNF in layers L4-L6 of peripheral cortex, and higher CNF in superficial layers (L1-L3). This analysis was conducted to verify that foveal and peripheral slices of $\mathrm{V} 1$ did not always vary by a global mean shift (potentially reflecting processing biases from AHBA). 


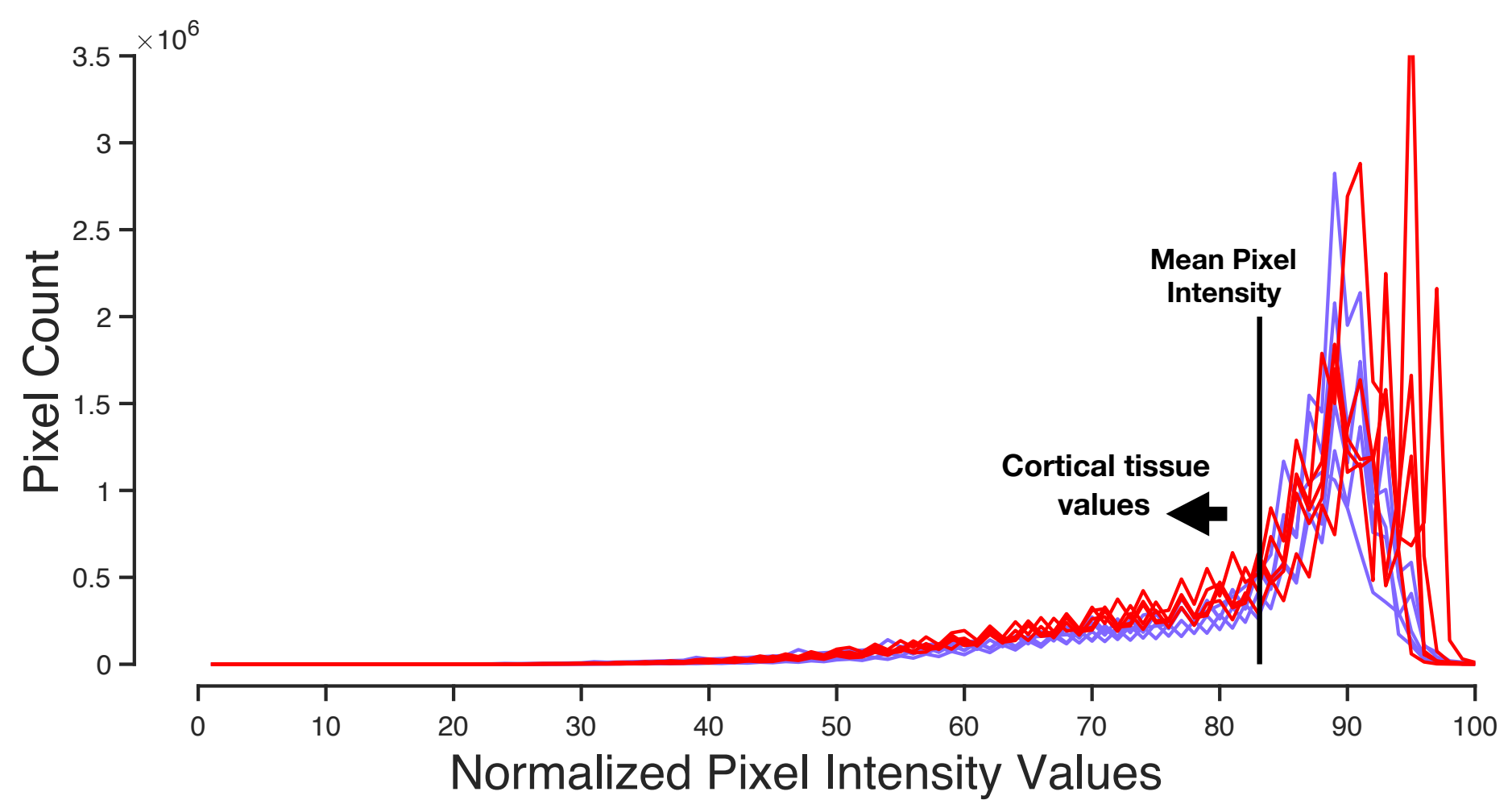

Supplementary Figure 8: Histograms of pixel values from foveal and peripheral Nissl stains of human tissue. To ensure that our chosen threshold for the BrainWalker CNF analysis was not impacting our results, we plotted the histogram of all pixel values from the foveal (red) and peripheral (purple) Nissl-stained slices from the AHBA. Higher values on the $x$-axis represent brighter pixels, with the large peaks from around $x=85$ to $x=100$ corresponding to the white background of the images, which represent the slide on which the tissue was mounted. The mean pixel intensity (the threshold used for the CNF analysis) is shown with a solid black line. The reader can appreciate that at the entire meaningful range $(>60)$ of the lower intensity values below the mean corresponding to cortical tissue, foveal slices (red) show higher values than peripheral (purple) slices. 
bioRxiv preprint doi: https://doi.org/10.1101/812560; this version posted October 21, 2019. The copyright holder for this preprint (which was not certified by peer review) is the author/funder, who has granted bioRxiv a license to display the preprint in perpetuity. It is made available under aCC-BY-NC-ND 4.0 International license.

Supplementary Table 1: Genes correlated with eccentricity. Positively correlated genes are shown in orange, negatively correlated in blue. The top $1 \%$ of genes (ranked by -log of $p$-value) is shown for each field map.

\begin{tabular}{|c|c|c|c|c|c|}
\hline V1 (Eccentricity) & V2 (Eccentricity) & V3 (Eccentricity) & V1 (Eccentricity) & V2 (Eccentricity) & V3 (Eccentricity) \\
\hline $\mathrm{ABCC} 1$ & $\mathrm{ABCC} 1$ & $\mathrm{~A} 1 \mathrm{CF}$ & ABHD6 & AARS2 & ABHD4 \\
\hline AC007743.1 & AC020663.1 & ABCA6 & AC005041.9 & AC139099.1 & AC010982.1 \\
\hline AC018865.8 & AC068353.1 & ABCC2 & AC034236.3 & ADAMDEC1 & AC015936.3 \\
\hline AC022498.2 & AC091878.1 & AC007952.2 & ACRV1 & AGAP11 & AC024270.1 \\
\hline AC068353.1 & AC100803.1 & AC018755.8 & ACSL5 & AMHR2 & $\mathrm{ADH} 1 \mathrm{C}$ \\
\hline AC134312.1 & AC112641.2 & AC091878.1 & ADAMTS16 & ANP32A & ADIG \\
\hline ACPT & ACTRT2 & ADCY1 & AKAP3 & AP006285.4 & ADRA1A \\
\hline ADAMTSL5 & ADAM23 & AFAP1 & ANGPT2 & ASB14 & AKIRIN2 \\
\hline ADD2 & ADCY1 & AFF1 & ANKS6 & ATAD3C & AP001372.1 \\
\hline ALG2 & ADCYAP1 & ALS2CR11 & ARHGEF17 & AVP & APOPT1 \\
\hline ANKLE1 & ADRA1D & ANKRD34C & ARL6IP5 & B4GALT1 & ASPG \\
\hline ANKRD20A7P & ALKBH8 & ARHGEF1 & ARL8A & BEST4 & BCAP29 \\
\hline ANKRD34C & ANKRD34C & ASPM & ARMC7 & BPNT1 & $\mathrm{BCHE}$ \\
\hline ARHGAP29 & ARC & ATP1B1 & ARMCX6 & BTN3A1 & BDKRB2 \\
\hline ARID1B & ARHGAP20 & ATP6V1G2 & ARRDC2 & BUD13 & BNIP1 \\
\hline ARID3A & ARSB & ATXN3 & ATF3 & C11orf53 & C10orf107 \\
\hline BIRC6 & ASAP1 & BBS7 & B2M & C11orf82 & C18orf51 \\
\hline BMPER & ASGR1 & BEGAIN & BACE1 & C12orf48 & C1orf187 \\
\hline BSND & ASH1L & BIRC6 & BACE2 & C15orf43 & C1orf93 \\
\hline BTBD11 & ASPSCR1 & BMPER & BTN3A1 & C15orf53 & C2orf28 \\
\hline BTC & ATG9B & C16ORF52 & BVES & C17orf89 & C3orf70 \\
\hline BUB1B & BDNF & C16orf78 & C10orf81 & C1orf122 & C5ORF27 \\
\hline C11orf84 & BIRC6 & C16orf92 & C11orf53 & C1orf168 & C6orf72 \\
\hline C14orf4 & BMPER & C22ORF45 & C12orf48 & C1orf194 & C7orf10 \\
\hline C1orf135 & BTBD11 & C3orf18 & C14orf91 & C1orf92 & C8orf71 \\
\hline C2orf54 & C10orf72 & C3orf35 & C19orf18 & C6orf118 & C9 \\
\hline C3orf59 & C12orf68 & C4orf22 & C19orf69 & C6orf195 & CARHSP1 \\
\hline C6ORF141 & C13orf18 & C7orf62 & C1QTNF6 & C6orf94 & CATSPER2P1 \\
\hline C8A & C13orf44 & CABC1 & C21orf37 & C6orf97 & CATSPERB \\
\hline C9orf71 & C17orf39 & CADPS & C5orf52 & C9orf163 & CCDC148 \\
\hline CAMK4 & C17orf80 & CALML4 & C6orf48 & C9orf96 & CCDC21 \\
\hline CASKIN1 & C17orf90 & CALML5 & C9orf117 & CAMP & CD63 \\
\hline CBLN4 & C1orf109 & CD163L1 & CARD18 & CARD18 & CDAN1 \\
\hline CCDC85B & C4orf22 & CD164L2 & CBR1 & CASS4 & $\mathrm{CDH} 9$ \\
\hline
\end{tabular}


bioRxiv preprint doi: https://doi.org/10.1101/812560; this version posted October 21, 2019. The copyright holder for this preprint (which was not certified by peer review) is the author/funder, who has granted bioRxiv a license to display the preprint in perpetuity. It is made available under aCC-BY-NC-ND 4.0 International license.

\begin{tabular}{|c|c|c|c|c|c|}
\hline V1 (Eccentricity) & V2 (Eccentricity) & V3 (Eccentricity) & V1 (Eccentricity) & V2 (Eccentricity) & V3 (Eccentricity) \\
\hline CD276 & CAMK4 & CD300LB & CCDC102B & CBWD6 & CLCA3P \\
\hline $\mathrm{CHIC1}$ & CBLN4 & $\mathrm{CDH} 2 \mathrm{O}$ & CCDC150 & CCDC21 & CLEC18A \\
\hline $\mathrm{CHML}$ & CCDC144NL & $\mathrm{CDH} 23$ & CCDC53 & CCDC74B & CLEC1A \\
\hline CHST7 & CD276 & CDKL4 & CD22 & CCDC90A & CLNS1A \\
\hline CITED2 & $\mathrm{CDH} 12$ & CDS1 & $\mathrm{CDH} 26$ & CCRL1 & COIL \\
\hline CLDN12 & $\mathrm{CDH} 23$ & CEACAM7 & CETP & ССТ6P1 & CPA2 \\
\hline CNKSR2 & $\mathrm{CDH} 7$ & CELA2B & CFB & $\mathrm{CDH} 26$ & CXorf58 \\
\hline COL4A1 & CDK5R1 & CGB2 & CHI3L2 & CFB & CYB5B \\
\hline $\mathrm{CRH}$ & CEBPG & CLCF1 & CHST6 & CFL1P1 & CYP2C9 \\
\hline CYB561D1 & CHAC2 & CLDND2 & CLDND1 & CHI3L2 & CYP4Z2P \\
\hline DAB1 & CHST8 & COASY & CLEC2A & CILP & DAB2 \\
\hline DNAJB13 & CITED2 & COL4A3 & CNN2 & CRYBA1 & DAZAP2 \\
\hline DTX4 & COL23A1 & COX10 & COMMD3 & CXorf58 & DFFA \\
\hline DUSP & COL5A2 & CRHR2 & CPB1 & CYP4B1 & DHODH \\
\hline DUSP6 & $\mathrm{CRH}$ & CRTC1 & CPSF2 & DCAF8L2 & DHX58 \\
\hline EGR3 & CRTC1 & CTRL & CRYBA1 & DDA1 & DIRC2 \\
\hline EIF2A & CRYBB1 & CTSZ & CSRP2 & DDX10 & DNASE1 \\
\hline ELANE & CS & DCLK1 & CUTA & DDX50 & DSE \\
\hline ELOVL3 & DBX1 & DIRAS1 & CYB5R2 & DEFB113 & EFHB \\
\hline ENPP5 & DIRAS1 & DLAT & CYR61 & DEFB123 & ERMAP \\
\hline ENTPD7 & DLEU7 & DOK3 & DBNDD2 & DIRC1 & EYA1 \\
\hline ERVV-1 & DNAJB5 & E2F2 & DDX43 & DNASE1L3 & FADD \\
\hline ETV3 & DPP6 & E2F8 & DEFB113 & DRD3 & FADS2 \\
\hline F2RL3 & DTX4 & EDEM3 & DEPDC7 & EDA & FAM108A5P \\
\hline FAM171A2 & DUSP6 & EIF3A & DMBT1 & EEF1A1P9 & FAM110C \\
\hline FAM190A & DUX4L15 & EME1 & DNASE1L3 & EFCAB11 & FAM111A \\
\hline FAM53A & EGFL7 & EMID2 & DUOX1 & ESF1 & FAM23A \\
\hline FOXA3 & EID3 & EPB41L5 & ECM2 & ESM1 & FAM75C1 \\
\hline FRG2C & ELAVL4 & FAM131C & EIF4ENIF1 & EYS & FASLG \\
\hline FRMD6 & EMB & FAM166A & ERBB3 & F11R & FER1L6 \\
\hline FXYD2 & ENPP5 & FBX016 & FAM112A & FAM120AOS & FIS1 \\
\hline G0S2 & ENTPD7 & FCF1 & FANCL & FAM129A & FLJ30679 \\
\hline GABRB2 & EPHA4 & FCRL1 & FBP2 & FAM160A1 & FLJ37396 \\
\hline GABRG3 & EPN1 & FGF20 & FCRL3 & FAM53C & FMO9P \\
\hline GALNT5 & EXTL3 & FLJ37201 & FLNB & FDPSL2A & FOXD3 \\
\hline GJB5 & FAM104A & FLJ42627 & FOLH1B & FEZF1 & FOXF2 \\
\hline
\end{tabular}


bioRxiv preprint doi: https://doi.org/10.1101/812560; this version posted October 21, 2019. The copyright holder for this preprint (which was not certified by peer review) is the author/funder, who has granted bioRxiv a license to display the preprint in perpetuity. It is made available under aCC-BY-NC-ND 4.0 International license.

\begin{tabular}{l|l|l|l|l} 
V1 (Eccentricity) V2 (Eccentricity) V3 (Eccentricity) & V1 (Eccentricity) & V2 (Eccentricity) & V3 (Eccentricity)
\end{tabular}

\begin{tabular}{|c|c|c|c|c|c|}
\hline GLTPD1 & FAM201B & FOXD4L4 & GAGE6 & FGA & GAL3ST2 \\
\hline GMEB2 & FBXL19-AS1 & FOXE3 & GALNTL2 & FLJ44635 & GALNT10 \\
\hline GPR135 & FOSB & GABRA1 & GEN1 & FLYWCH2 & GCNT2 \\
\hline GPR156 & FSTL4 & GAL3ST3 & GMPR2 & FOXB1 & GLOD4 \\
\hline GRIP1 & FTSJ2 & GALNT8 & GNL3 & GCSH & GNG10 \\
\hline GRM2 & FUT11 & GPA33 & GNRHR2 & GHSR & GPC6 \\
\hline GRM7 & GABRB2 & GPR157 & GOT1L1 & GIMAP2 & GUK1 \\
\hline H2AFB2 & GABRD & GPR26 & GP6 & GLTPD2 & GYG2 \\
\hline H2AFY2 & GAL3ST3 & GVINP1 & GPR18 & GOT1L1 & H3F3B \\
\hline HCN3 & GFRA2 & HAAO & GPX8 & GRIN3B & HEXA \\
\hline HCRTR1 & GJB5 & $\mathrm{HCN} 1$ & H3.Y & GSG2 & HEY1 \\
\hline HCRTR2 & GLCE & HCRTR1 & HHLA3 & HAUS8 & HFM1 \\
\hline HDAC9 & GLT25D1 & HERC6 & HOXC10 & HLA-J & HLA-DQA1 \\
\hline HIST1H2AE & GPR158 & HIVEP1 & HSPA1A & HMGN2P15 & HNRNPR \\
\hline HIST1H3D & GRASP & HIVEP2 & IGFBP1 & HMHB1 & $\mathrm{HYI}$ \\
\hline HIST1H4K & GRIP1 & HPX & IL15 & HMX3 & IL24 \\
\hline HIST4H4 & GRM2 & HS6ST2 & IL1RL1 & HNRNPA1P12 & IL4R \\
\hline HLA-DPB2 & GRM7 & HSD11B2 & IL24 & HOXC4 & IMPDH2 \\
\hline HLF & H2AFB2 & HSF1 & IL4R & HTA & ISOC1 \\
\hline HOXC11 & HDAC8 & HSPA4L & INHA & $\mathrm{IFI} 27$ & JUN \\
\hline IPMK & HIST1H1E & HUNK & ITGA8 & IFITM3 & KHDRBS2 \\
\hline IPO4 & HIST1H3F & HYLS1 & ITIH5 & IFNA1 & KLRC3 \\
\hline IRF1 & HIST1H4F & IGF2BP1 & KIF7 & IFT74 & LACTB2 \\
\hline IRX3 & HIST1H4J & $\mathrm{IHH}$ & KLHL4 & IL24 & LCN12 \\
\hline KCNA6 & HIST1H4K & IL7R & KRT10 & IL4R & LETM1 \\
\hline KCNB2 & HIVEP2 & ITGA11 & KRT8P45 & IQSEC2 & LGI4 \\
\hline KCNG2 & HTR3B & JMY & LACTB2 & ISX & LIG1 \\
\hline KCNG4 & HTR5A & KBTBD8 & LAYN & ITGA1 & LINC00471 \\
\hline KCNH5 & INA & KIAA0408 & LIPA & ITGB2 & LOC100128009 \\
\hline $\mathrm{KCNJ} 14$ & IP6K1 & KIAA1009 & LIPJ & ITIH5 & LOC100131510 \\
\hline KCNJ16 & JMJD5 & KLHL26 & LOC100130262 & IVL & LOC100292680 \\
\hline KCTD9P2 & KCNA1 & KLHL6 & LOC100130344 & KIF20A & LOC283861 \\
\hline KDELC2 & KCNA6 & LCE3C & LOC100130932 & KRTAP19-8 & LOC389787 \\
\hline KIAA1161 & KCND3 & LCN6 & LOC100287869 & LAMC2 & LOC441204 \\
\hline KIAA1530 & KCNG2 & LGMN & LOC100289070 & LINC00116 & LOC642943 \\
\hline KIR3DL3 & KCNH5 & LHB & LOC100294396 & LINC00608 & LOC645212 \\
\hline
\end{tabular}


bioRxiv preprint doi: https://doi.org/10.1101/812560; this version posted October 21, 2019. The copyright holder for this preprint (which was not certified by peer review) is the author/funder, who has granted bioRxiv a license to display the preprint in perpetuity. It is made available under aCC-BY-NC-ND 4.0 International license.

\begin{tabular}{l|l|l|l|l} 
V1 (Eccentricity) V2 (Eccentricity) V3 (Eccentricity) & V1 (Eccentricity) & V2 (Eccentricity) & V3 (Eccentricity)
\end{tabular}

\begin{tabular}{|c|c|c|c|c|c|}
\hline KLC3 & KCNS2 & LILRB3 & LOC653653 & LIPJ & LOC645431 \\
\hline KRTAP1-4 & KCTD8 & LILRB4 & LOC728533 & LOC100130935 & LOC729859 \\
\hline KRTAP22-1 & KCTD9P2 & LIN28B & LOC728673 & LOC100131727 & LOC731157 \\
\hline KRTAP4-5 & KIAA0748 & LINC00176 & LOC728819 & LOC100132658 & LOH12CR1 \\
\hline LFNG & KIAA1161 & LIPT1 & LOC732272 & LOC100133775 & LSM5 \\
\hline LIN7C & KIAA1632 & LOC100131471 & LOXL4 & LOC100287017 & MAPK3 \\
\hline LINC00339 & KLF9 & LOC100289944 & LRRIQ4 & LOC100287090 & MEXЗА \\
\hline LINC00545 & KLHL11 & LOC100290497 & LTF & LOC100293158 & MGC34034 \\
\hline LOC100131830 & KRT16P2 & LOC149837 & MAK & LOC100294396 & MIS12 \\
\hline LOC100133299 & KRT18P55 & LOC200726 & MAMDC2 & LOC285741 & MMP2 \\
\hline LOC100287391 & LGALS7 & LOC282997 & MCM2 & LOC388630 & MRPL24 \\
\hline LOC100289632 & LHFPL2 & LOC388849 & MGC12916 & LOC441124 & MS4A15 \\
\hline LOC100291206 & LIG3 & LOC401052 & MITF & LOC442293 & MTF1 \\
\hline LOC100291630 & LINC00340 & LOC440461 & MORC4 & LOC493754 & NECAP2 \\
\hline LOC284072 & LINC00473 & LOC645944 & MS4A6A & LOC550643 & NEK11 \\
\hline LOC645431 & LIPT1 & LOC728613 & MYOT & LOC554207 & NUDT21 \\
\hline LOC652614 & LOC100132364 & LYVE1 & NANOGP8 & LOC642103 & OR10W1 \\
\hline LOC728056 & LOC100133299 & MDGA2 & NDE1 & LOC650095 & OR13C3 \\
\hline LOC728566 & LOC100289550 & MED23 & NEK3 & LOC728392 & $\mathrm{OR} 13 \mathrm{H} 1$ \\
\hline LONRF3 & LOC100291124 & MGC16025 & NENF & LOC728673 & OR2H2 \\
\hline LRRK1 & LOC652614 & MIA3 & NMB & LOC729046 & OR5111 \\
\hline LYN & LOC84989 & MTDH & NMNAT1 & LOC729739 & OR5B3 \\
\hline MORN1 & LRRC61 & MYO5B & NSBP1 & $\mathrm{LRCH} 4$ & PARD3 \\
\hline MSX1 & LRRC8D & NDFIP2 & NUF2 & LRRIQ4 & PCBD2 \\
\hline MUCL1 & LRRK1 & NR1I3 & OIP5 & MAFG-AS1 & PCDH21 \\
\hline MYD88 & LYPD5 & OR1L6 & OMG & MCART6 & PGPEP1 \\
\hline MYO1B & MAD2L1 & OR51B5 & OPALIN & MRGPRF & PHKG1 \\
\hline NANOS2 & MAFF & OR5A1 & OR10X1 & MYPN & PLAC8L1 \\
\hline NAPRT1 & MAL2 & OR8J1 & PADI6 & NACAP1 & POLR2I \\
\hline NOTUM & MAP2K7 & OSBPL3 & PARD6G & NCR2 & PON3 \\
\hline NRTN & MARCH11 & PAG1 & PARP3 & NDUFS5P7 & PPAT \\
\hline OCLM & MCF2L-AS1 & PCDHGB3 & PARP4 & NEUROG3 & PPIL6 \\
\hline OPRD1 & MKS1 & PCSK4 & PDE11A & NFYB & PRDX1 \\
\hline OR10V1 & MMAA & PDC & PDE6B & NME3 & PRRG3 \\
\hline OR13C8 & NRTN & PDE10A & PEBP1 & NOD1 & PSG2 \\
\hline OR13H1 & OLFM3 & PDE7A & PHACTR4 & OR5L2 & PSIP1 \\
\hline
\end{tabular}


bioRxiv preprint doi: https://doi.org/10.1101/812560; this version posted October 21, 2019. The copyright holder for this preprint (which was not certified by peer review) is the author/funder, who has granted bioRxiv a license to display the preprint in perpetuity. It is made available under aCC-BY-NC-ND 4.0 International license.

\begin{tabular}{l|l|l|l|l} 
V1 (Eccentricity) V2 (Eccentricity) V3 (Eccentricity) & V1 (Eccentricity) & V2 (Eccentricity) & V3 (Eccentricity)
\end{tabular}

\begin{tabular}{|c|c|c|c|c|c|}
\hline OR1N1 & ONECUT3 & PGC & Pl16 & ORM1 & PUF60 \\
\hline OR2A14 & OPRD1 & PGR & PIGT & PABPC3 & QPRT \\
\hline $\mathrm{OR} 2 \mathrm{H} 2$ & OR2M5 & PLCL2 & PIWIL3 & PARP4 & RAB36 \\
\hline OR4D6 & OSBPL10 & PLEKHG6 & PKD1L3 & PCGF2 & RAD54L \\
\hline PABPC1L2B & PABPC1L2A & PNP & PLEKHG3 & PGAM4 & $\mathrm{RDH} 8$ \\
\hline PCDHB18 & PAH & POU6F2 & POPDC2 & PHC3 & REG1P \\
\hline PCDHGA9 & PCDHA5 & PPM1E & PPIA & PIWIL3 & RFT1 \\
\hline PCNXL3 & PDE10A & PRRG4 & PRKCH & POF1B & RGS21 \\
\hline PDE10A & PDP2 & PRRT3 & PROM1 & PPBPL2 & $\mathrm{RHOA}$ \\
\hline PLA2G2D & PHF20 & PRSS27 & PRR20 & PPIAL4G & RIT1 \\
\hline PLCH2 & PI4K2A & PTK7 & PTP4A2 & PROM1 & RNASE4 \\
\hline PLEKHF2 & PIAS3 & PUS10 & PUS7L & PRRX1 & RP11-169K16.9 \\
\hline PLVAP & PINX1 & RAB3GAP2 & QDPR & PTGIR & RP11-193H5.1 \\
\hline PPAPR5 & PLCB4 & RAl2 & RBP3 & PXDN & RP11-428G5.1 \\
\hline PPP1R3G & PPP1R13B & REEP5 & RBP7 & QKI & RP11-453F18_B.1 \\
\hline PPYR1 & PRDM1 & REG1B & RCL1 & RAD51L1 & RP11-535K1.1 \\
\hline PRB3 & RASGRF2 & RIMS3 & RESP18 & RAN & RPL13 \\
\hline PRDM1 & RASSF5 & RNASE2 & RIBC2 & RP1-10C16.2 & RPL18 \\
\hline PRR5 & RCSD1 & RNF215 & RNF183 & RP11-193H5.1 & RPS24 \\
\hline PRSS22 & RG9MTD1 & RP11-206L10.11 & RNF39 & RP11-349A22.5 & RPS27L \\
\hline RAPH1 & RIMS3 & RP13-254B10.1 & RP11-115C21.2 & RP11-379K17.4 & RUNX3 \\
\hline RGR & SBK1 & RP5-1187M17.1 & RP11-45B20.3 & RP11-475E11.5 & $\mathrm{S} 100 \mathrm{~A} 13$ \\
\hline RHBDD3 & SCN5A & SAMD5 & RP11-537P22.1 & RPL23AP82 & SCPEP1 \\
\hline ROD1 & SF3B4 & SERINC4 & RP3-486B10.1 & RPL24 & SDF2L1 \\
\hline RP13-254B10.1 & SHQ1 & SH3D20 & RPL19P12 & RPL32 & $\mathrm{SH} 2 \mathrm{D} 4 \mathrm{~B}$ \\
\hline RP5-1187M17.1 & SIDT1 & SH3RF3-AS1 & RPL23AP82 & RPLP1 & SHISA4 \\
\hline RPL32P3 & SIPA1L2 & SLAMF8 & RSU1 & RPS18 & SIPA1 \\
\hline RPUSD1 & SLC2A6 & SLC16A3 & SCGB1D4 & S100A10 & SIX2 \\
\hline S100A5 & SLC37A2 & SLC17A9 & SDCCAG3P2 & SEMG2 & SLC22A24 \\
\hline SARDH & SLITRK4 & SLC37A2 & SEMA3C & SERBP1 & SLC27A5 \\
\hline SBF1P1 & SNX12 & SLC4A9 & SGK1 & SESTD1 & SLC6A13 \\
\hline SCN5A & SPATS2 & SLC7A5P2 & SH3BP4 & SET & SLC6A2 \\
\hline SENP8 & SPIRE1 & SLC9A1 & SH3PXD2A & SH3BP4 & SLC7A9 \\
\hline SERTAD1 & ST8SIA5 & SLFNL1 & SLC26A9 & SIM2 & SNRNP27 \\
\hline SHROOM3 & STK40 & SMPD3 & SLC7A5 & SLC22A18AS & SSX2B \\
\hline SLC22A1 & STS & SMPX & SLC9A9 & SLC6A3 & STK16 \\
\hline
\end{tabular}


bioRxiv preprint doi: https://doi.org/10.1101/812560; this version posted October 21, 2019. The copyright holder for this preprint (which was not certified by peer review) is the author/funder, who has granted bioRxiv a license to display the preprint in perpetuity. It is made available under aCC-BY-NC-ND 4.0 International license.

\begin{tabular}{|c|c|c|c|c|c|}
\hline V1 (Eccentricity) & V2 (Eccentricity) & V3 (Eccentricity) & V1 (Eccentricity) & V2 (Eccentricity) & V3 (Eccentricity) \\
\hline SLC22A13 & STXBP5L & SNX25 & SMOC1 & SLC9A11 & STMN4 \\
\hline SLC25A2 & SUPT16H & SOST & SNIP1 & SLC9A3 & STXBP6 \\
\hline SLC36A1 & SYT12 & SSU72P8 & SNX29 & SNIP1 & SULT1E1 \\
\hline SLC41A2 & TAPBP & ST8SIA5 & SPANXA1 & SNPH & SYNJ2BP \\
\hline SLC5A5 & TBX21 & TCF7L2 & SPIC & SPEF2 & TELO2 \\
\hline SNAl1 & TCERG1L & TEX9 & SPINK4 & SPINK4 & TEX264 \\
\hline SPDYE2 & TCTN2 & TNFRSF4 & SPINT4 & SPINT4 & TLCD2 \\
\hline SPNS3 & TM6SF1 & TPTE2P1 & SPTA1 & SPOCK2 & TM7SF2 \\
\hline STK3 & TMEM25 & TRAF5 & TCFL5 & SPRR1B & TMEM11 \\
\hline SYT12 & TPBG & TRGV5 & TMEM194B & STRA6 & TMEM27 \\
\hline TCTN2 & TSPAN5 & TRIM21 & TMEM206 & SUMO2 & TMEM47 \\
\hline TET3 & ТТСЗ0А & TSKS & TNFAIP6 & T & TMPRSS6 \\
\hline TIMP3 & TUBA4B & TSPAN5 & TRADD & TNF & TNFRSF10C \\
\hline TNFRSF12A & UBASH3B & ТТСЗ9А & TRIM38 & TRAM1 & TRHR \\
\hline TNFSF9 & UBTD2 & U2AF2 & TTLL5 & TRMT5 & TSPAN4 \\
\hline ТTC24 & UHRF1 & UGP2 & TUBB1 & TUSC1 & TXNDC6 \\
\hline UBASH3B & USP10 & UHRF1 & TYMS & TXNL4A & UCKL1 \\
\hline UBTD2 & UST & UHRF1BP1L & UBA6 & UBOX5 & ULK4 \\
\hline UHRF1 & VIP & ZDHHC2 & USP17L1P & UFM1 & USP17L1P \\
\hline WDR81 & VWC2L & ZDHHC8 & WDR49 & UQCRHP2 & USP18 \\
\hline XIST & WARS2 & ZNF233 & WNK1 & USP54 & VAT1 \\
\hline ZBTB5 & ZBTB5 & ZNF257 & WTH3DI & VAX2 & WAS \\
\hline ZBTB7C & ZDHHC8 & ZNF287 & YWHAQ & WDR49 & WDR6 \\
\hline ZDHHC8 & ZNF35 & ZNF354B & ZBED5 & WTH3DI & WFIKKN2 \\
\hline ZNF239 & ZNF385D & ZNF385A & ZDHHC15 & XIRP1 & YES1 \\
\hline ZNF385D & ZNF48 & ZNF408 & ZNF251 & ZCRB1 & YIPF7 \\
\hline ZNF697 & ZNF567 & ZNF700 & ZNF311 & ZNF727 & ZBTB8B \\
\hline ZNF772 & ZNF74 & ZNF823 & ZNF705C & ZNF85 & ZDHHC8P1 \\
\hline ZSCAN22 & ZSCAN22 & ZNHIT2 & ZWINT & ZWINT & ZFP30 \\
\hline
\end{tabular}


Supplementary Table 1 (continued): Genes correlated with polar angle. Positively correlated genes are shown in orange, negatively correlated in blue. The top $1 \%$ of genes (ranked by -log of $p$-value) is shown for each field map.

\begin{tabular}{|c|c|c|c|c|c|}
\hline V1 (Polar angle) & V2 (Polar angle) & V3 (Polar angle) & V1 (polar angle) & V2 (polar angle) & V3 (polar angle) \\
\hline AC008429.1 & ABHD12B & ABHD14B & AC005077.3 & $\mathrm{ABCA} 7$ & AC002472.1 \\
\hline AC011346.1 & AC008429.1 & $\mathrm{ABI} 1$ & AC005840.2 & AC008537.3 & AC087392.3 \\
\hline AC015871.2 & AC013271.3 & AC008073.2 & AC103965.1 & AC103965.1 & ACTRT2 \\
\hline AC068181.1 & AC063965.2 & ACCN1 & AC106739.1 & ACAP3 & AKAP11 \\
\hline AC092327.1 & AC068181.1 & ADAMTS9 & ACAP3 & ACLY & ANXA9 \\
\hline ACOT6 & AC084209.1 & $\mathrm{ADH} 1 \mathrm{C}$ & $\mathrm{ACHE}$ & ACTRT2 & AP3B1 \\
\hline ACRV1 & ACOT6 & AKIRIN2-AS1 & ACIN1 & AFF3 & APOBEC3G \\
\hline ADAD2 & ACTA2 & ALDOB & ADAM15 & AHDC1 & APPBP2 \\
\hline ADAMTS18 & ACTG2 & ANKRD1 & ADD2 & AMDHD2 & ARFGEF1 \\
\hline ADCY10 & ADAMTS2 & ANXA2P1 & AHDC1 & ANKRD20A7P & ARMC3 \\
\hline AGTR1 & AIM2 & AOC3 & ALKBH6 & ARR3 & ARSB \\
\hline ANKRD30A & ALPK3 & ATG16L2 & ANAPC13 & ART5 & ASB4 \\
\hline ATF3 & ANGPT1 & ATP5E & ANKRD24 & ASH1L & ATF7IP \\
\hline ATOH1 & AOC3 & ATP9B & AP1G2 & ATXN3 & BBX \\
\hline BEND3 & ASPN & BBS1 & ARFGAP1 & AURKC & BEND4 \\
\hline BSX & ATAD3C & BX248398.1 & ATCAY & BANF1 & BIRC6 \\
\hline BX255925.2 & ATF7 & C10orf10 & ATP8B2 & BCL11B & BPGM \\
\hline C10orf53 & ATP11C & C10orf104 & ATXN1 & BEND5 & BZW1 \\
\hline C11orf34 & AVIL & C10orf26 & AURKA & BIRC6 & C14orf119 \\
\hline C12orf48 & C10orf68 & C10orf68 & BBC3 & C10orf55 & C19orf40 \\
\hline C14orf54 & C15orf29 & C11orf17 & BCL6 & C19orf52 & C1orf156 \\
\hline C1orf180 & C1orf229 & C11orf57 & C11orf63 & C1orf190 & C1orf55 \\
\hline C20orf29 & C3orf15 & C11orf75 & C15orf38 & C1orf68 & C2orf61 \\
\hline C3orf44 & C3orf20 & C19orf73 & C1orf190 & C20orf144 & C4orf37 \\
\hline C3orf48 & C5orf25 & C1orf204 & C4orf22 & C7ORF74 & C5orf47 \\
\hline C5orf25 & C6orf142 & C1orf51 & CACNA1A & CA12 & C6orf153 \\
\hline C5orf52 & C6orf15 & C3orf31 & CCDC136 & CABP7 & C6orf182 \\
\hline C6orf126 & $\mathrm{C} 7$ & C6orf26 & CCDC142 & CALHM1 & C6orf91 \\
\hline C9orf117 & CA13 & C6orf48 & CCDC57 & CBX6 & C7orf70 \\
\hline CARD18 & CAPN14 & C8orf59 & CCDC66 & CBX8 & CALCB \\
\hline CASR & CCDC102A & CAMK2A & CD276 & CD276 & CDC20 \\
\hline CCDC113 & CCDC113 & CAV1 & CEND1 & CDC2L1 & CDC42BPG \\
\hline CCDC135 & CCDC18 & CAV2 & CEP152 & CDC37 & $\mathrm{CDH} 20$ \\
\hline CCDC140 & CCDC46 & CBR3 & CERK & CDCA5 & CDK7 \\
\hline CCDC27 & CCDC52 & CCDC102A & CHD6 & CHD3 & CDY2A \\
\hline CCL16 & CCL2 & CCDC13 & CHRD & COL23A1 & CHEK1 \\
\hline CCR8 & CD163 & CCDC46 & $\mathrm{ClZ1}$ & CREB3 & CLCN3 \\
\hline
\end{tabular}




\begin{tabular}{|c|c|c|c|c|c|}
\hline V1 (Polar angle) & V2 (Polar angle) & V3 (Polar angle) & V1 (polar angle) & V2 (polar angle) & V3 (polar angle) \\
\hline CD177 & CD2AP & CDCA4 & COL18A1 & CTNNBIP1 & CLDND2 \\
\hline $\mathrm{CDH} 1$ & $\mathrm{CDH} 18$ & CDKL3 & CREBL1 & CWC15 & CRYBG3 \\
\hline CDNF & CDSN & CDKN2C & CRKL & DND1P1 & CSNK2A1 \\
\hline CDRT15 & CENPH & CFHR2 & CUL7 & DRD4 & DCTN4 \\
\hline CETP & CENPK & CLIC1 & CWC15 & DSC2 & DDC \\
\hline CLRN3 & CHMP4C & CLSTN1 & CYB561D1 & EGR3 & DDI2 \\
\hline CRISP1 & CHRNA3 & CMTM2 & CYP11A1 & EMX2OS & DNAH9 \\
\hline CXCL11 & CHRNA6 & CNN1 & DAXX & ERBB2 & DOCK1 \\
\hline CXCL9 & CLUL1 & CNPY2 & DCX & $\mathrm{F} 7$ & DOCK2 \\
\hline CXXC4 & COL13A1 & CNTLN & DNAJC11 & FAM129C & DSC2 \\
\hline CYAT1 & COL14A1 & COL1A1 & EBF4 & FAM74A1 & DTX4 \\
\hline CYLC2 & COL1A1 & COL4A2 & ENSA & FEN1 & E2F8 \\
\hline CYP3A5 & COL4A2 & СРАЗ & ERG & FITM2 & EFTUD1 \\
\hline CYP4F24P & CPXM2 & CPSF4 & ERVV-1 & FKBP8 & ELOVL2 \\
\hline DCAF4 & CSN3 & CREB3L2 & ETV3 & FOXA3 & EVX2 \\
\hline DDX28 & CXCL9 & CTAG1B & FAM120B & G6PC3 & FABP4 \\
\hline DDX43 & CXCR3 & CTBP1 & FAM153A & GMCL1P1 & FAM159A \\
\hline DDX50 & CYP4B1 & CXCR3 & FASN & GMIP & FAM166A \\
\hline DIAPH3 & DCAF4 & CYR61 & FKBP8 & GNMT & FCF1 \\
\hline DNTT & DDX50 & DGCR9 & FLJ36848 & GPR173 & FCRL1 \\
\hline DUPD1 & DEPDC1B & $\mathrm{DHODH}$ & FLJ42627 & GRIPAP1 & FGFR1 \\
\hline EN1 & DGAT1 & DIO3OS & FOXO3 & GRPEL1 & FNIP2 \\
\hline EPHA1 & DHX29 & DSE & FXYD2 & GSX2 & FOLR1 \\
\hline ESR2 & DPT & ECSCR & GDAP2 & H2AFB2 & GALNT8 \\
\hline EVC & DSC1 & EEF1A1P24 & GEMIN7 & HAAO & GRM8 \\
\hline FABP12 & DSCAML1 & EEF1A1P9 & GJA9 & HAND2 & GRTP1 \\
\hline FAM120AOS & DSP & FAM111A & GLTPD1 & HBBP1 & GSC2 \\
\hline FAM124B & ECM2 & FAM120AOS & GNPDA1 & HDLBP & H2AFB1 \\
\hline FAM154A & ECSCR & FAM156A & GPR156 & HEATR1 & HCG26 \\
\hline FAM71B & ELL & FBLIM1 & GPR20 & $\mathrm{HRG}$ & HCP5B \\
\hline FANCL & ELN & FBLN5 & GPR21 & $\mathrm{HRH} 1$ & HIPK3 \\
\hline FCN2 & EP400NL & FKBP10 & GPRIN3 & HSD17B1 & HIST1H3A \\
\hline FCRL2 & EPHA1 & FOLR4 & GRPEL1 & IGLL1 & HIST1H3F \\
\hline FER1L6 & EPHA5 & FOXK1 & GTPBP5 & IL22RA2 & HIST1H4G \\
\hline FOXI2 & EYS & FOXL1 & HDAC8 & IL26 & HIST1H4J \\
\hline FRMD3 & FAM129A & FRMD3 & HIST1H1C & INO80B & HMMR \\
\hline GAGE6 & FAM186B & GNG11 & HIST1H1E & KCNJ14 & HOXA4 \\
\hline GATA2 & FANCL & GNPDA2 & HIST4H4 & KIAA1161 & HSPA5 \\
\hline GATA3 & FBXO4 & GPR120 & HM13 & KIAA1522 & ICOSLG \\
\hline GBP5 & FHL5 & GPX8 & HMGB1P1 & KIAA1530 & IL15RA \\
\hline
\end{tabular}




\begin{tabular}{|c|c|c|c|c|c|}
\hline V1 (Polar angle) & V2 (Polar angle) & V3 (Polar angle) & V1 (polar angle) & V2 (polar angle) & V3 (polar angle) \\
\hline GJC3 & FLNA & GRAMD4 & HNRNPUL2 & KIAA1549 & INTS12 \\
\hline GLYATL1P2 & FOXC1 & HAUS7 & HSPB6 & KLC3 & IRX2 \\
\hline GNAT1 & FOXN1 & HMCN1 & IFIT3 & KRTAP4-5 & KAAG1 \\
\hline GOT1L1 & FRZB & HMGN2 & IGSF9 & L1CAM & KCNA10 \\
\hline GRHL1 & GAL3ST2 & HNRNPAO & INTS4L1 & LCE1F & KCNG2 \\
\hline GSN-AS1 & GATA2 & HYAL2 & ITPR1 & LCN1 & KCNJ15 \\
\hline GUSBP10 & GCET2 & ICAM1 & KCNC3 & LEO1 & KCTD9P2 \\
\hline HES2 & GOT1L1 & $\mathrm{IFIH} 1$ & KCNJ14 & LGALS7 & KDELC2 \\
\hline HIST1H2BA & GPX8 & IGFBP4 & KCNJ8 & LIG3 & KIAA1324L \\
\hline HPD & GTSF1 & IGFBP7 & KCTD1 & LILRA3 & KIAA1530 \\
\hline HPGD & GYS1 & IQUB & KCTD8 & LMOD2 & KLF17 \\
\hline HSF2BP & HAUS8 & ITGA8 & KDM5D & LOC100287282 & KPNA3 \\
\hline IFNK & HRC & ITGB1BP2 & KGFLP2 & LOC100288972 & KRT76 \\
\hline IGHG4 & ICAM2 & JAG1 & KIAA1522 & LOC100289550 & KSR1 \\
\hline IGSF2 & IGHG2 & KRTAP5-4 & KIAA1530 & LOC100290375 & L1CAM \\
\hline IL4 & INO80D & LINC00307 & KIR3DL3 & LOC100291124 & LDHAL6B \\
\hline ISLR2 & ITGA4 & LINC00338 & KLC3 & LOC392264 & LELP1 \\
\hline ITGB6 & ITGA8 & LOC100130700 & KLC4 & LOC400406 & LGl1 \\
\hline KIF24 & JAG1 & LOC100130935 & KRTAP4-5 & LOC400419 & LIG3 \\
\hline KLHDC7B & KCNU1 & LOC100288618 & KRTAP4-8 & LOC400590 & LINC00176 \\
\hline KRT13 & KIAA0040 & LOC100289058 & KRTAP5-11 & LOC643750 & LINGO4 \\
\hline KRT23 & KIAA1524 & LOC100289525 & LDB1 & LOC646043 & LMLN \\
\hline KRT27 & KLHL14 & LOC100294396 & LEO1 & LOC727803 & LOC100130000 \\
\hline KRT73 & KLHL28 & LOC440084 & LOC100133299 & LOC728056 & LOC100130811 \\
\hline KRT77 & LAMC3 & LOC441204 & LOC100287825 & LOC728543 & LOC100287987 \\
\hline LINC00421 & LINC00421 & LOC644397 & LOC100288583 & LOC728606 & LOC100292160 \\
\hline LIPJ & LIPC & LOC653888 & LOC100288972 & LOC729887 & LOC100294033 \\
\hline LOC100129852 & LOC100129166 & LOC729973 & LOC100289550 & LOC730183 & LOC400406 \\
\hline LOC100130071 & LOC100133862 & LOC732327 & LOC158696 & LRRC4B & LOC402377 \\
\hline LOC100132738 & LOC100287090 & LOH12CR1 & LOC284072 & LRRTM2 & LOC441528 \\
\hline LOC100133153 & LOC100293158 & LRRC47 & LOC389842 & LTB4R2 & LOC651959 \\
\hline LOC100287487 & LOC100293904 & LSM5 & LOC391334 & MAML3 & LOC728543 \\
\hline LOC100288908 & LOC100507637 & LTBP1 & LOC392264 & MAN1B1 & LOC728853 \\
\hline LOC100291229 & LOC439914 & LTBP4 & LOC401321 & MAPK12 & LRRC3 \\
\hline LOC220906 & LOC440173 & MDFIC & LOC727803 & MAPT-AS1 & LRRC45 \\
\hline LOC646762 & LOC645722 & MED17 & LPCAT4 & MEPE & MAP2K5 \\
\hline LOC646934 & LOC646509 & MGC24103 & LRP1 & MGC39584 & MFI2 \\
\hline LOC653203 & LOC646762 & MORC2 & LRRK1 & MIXL1 & MGC16025 \\
\hline LOC732327 & LOC729839 & MRGPRF & MUL1 & MRPL50 & MIR17HG \\
\hline LY6G6D & LOC732327 & MRPL43 & MYD88 & MVK & MMGT1 \\
\hline
\end{tabular}




\begin{tabular}{|c|c|c|c|c|c|}
\hline V1 (Polar angle) & V2 (Polar angle) & V3 (Polar angle) & V1 (polar angle) & V2 (polar angle) & V3 (polar angle) \\
\hline LYZ & LRRC67 & MSH5 & NAPRT1 & MYBPH & MTDH \\
\hline MAGEA4 & LTBP1 & MSL3 & NES & NANOS2 & MTMR3 \\
\hline MAGEB1 & MALT1 & MTHFR & NEU4 & NAPRT1 & MTNR1B \\
\hline MAGOH & MAP4K1 & MTND4P12 & NFATC2 & NAT2 & NAG18 \\
\hline MEP1A & MARVELD1 & MUSTN1 & NGRN & NLRP2 & NALCN \\
\hline MIA2 & MCCC1 & MYL12A & $\mathrm{OGDH}$ & NUCB1 & NAPSA \\
\hline MKI67 & MCM2 & MYL9 & OLR1 & OGDH & NAT8B \\
\hline MKRN7P & MDFIC & NDUFB8 & OR1N1 & OR1K1 & NCBP1 \\
\hline MMP1 & MFAP4 & NFYB & ORMDL3 & OR2T5 & NEBL \\
\hline MMP20 & MPP4 & NLRP3 & OTX1 & OR4D5 & NFATC4 \\
\hline MORC4 & MRGPRF & NR4A1 & PCIF1 & OR4M2 & NIPA2 \\
\hline MOV10L1 & MYLK4 & NRP1 & PDE3B & OR5K1 & NLGN2 \\
\hline MTNR1B & NANOGP8 & OAZ1 & PILRA & ORM2 & NLRP10 \\
\hline MYLK3 & NAP1L6 & OGN & PINX1 & PAPLN & NLRP4 \\
\hline NEK5 & NDFIP2 & OPN1SW & PIP5K1C & PCYOX1L & NR1I3 \\
\hline NKX3-2 & NFYB & ORAI1 & PLXNA3 & PDE3B & OCRL \\
\hline NUF2 & NFYC & PARD6G & POMZP3 & PIK3AP1 & OR10A4 \\
\hline OR10G9 & NHLRC2 & PCDHB6 & PP13 & PIK3R2 & OR2T33 \\
\hline OR1J1 & NKAPL & PDCD2L & PPFIA4 & PINX1 & OR8J1 \\
\hline OR52V1P & NOBOX & PDGFRB & PPP1R3G & PLD2 & ORM2 \\
\hline OR5J2 & NSBP1 & PDZRN3 & PRB4 & PLEC1 & OVOL2 \\
\hline OR8J3 & NT5C1A & PFAS & PRKACA & PLGLB2 & PAX5 \\
\hline OR8K1 & NUP188 & PGM3 & PRPF40B & PLXNA3 & PBLD \\
\hline ОTC & OGN & PIP5K1A & PRR16 & POLR3A & PCDHGA11 \\
\hline OTOR & OLFML2A & PKIG & PVT1 & POPDC3 & PCDHGA9 \\
\hline PADI6 & OMD & PLAC8L1 & RETN & POU2F2 & PDK3 \\
\hline PAGE2B & OR5H1 & PLAT & RHBDD3 & PRAMEF7 & PFKFB4 \\
\hline PAX8 & OR5V1 & PROCR & RHOBTB1 & PRB4 & PLA2G5 \\
\hline PDCL2 & OTUD1 & PRPS2 & RHOBTB2 & PRCC & РОТЕН \\
\hline PDZK1IP1 & PARD6G & PTGIS & RHOQP3 & PRG3 & POU1F1 \\
\hline PIK3C2G & PCDHB5 & PXDN & RNF144A & PRSS22 & POU2F3 \\
\hline PLUNC & PDE12 & RAMP3 & RNF157 & PTCH1 & PRAME \\
\hline POM121 & PGF & RANBP3 & RNPS1P1 & PVT1 & PRAMEF25 \\
\hline PRG2 & PIK3C2G & RASL12 & RP11-440L14.1 & RBM22 & PRKG2 \\
\hline PSG11 & PLN & RBPMS & RP5-1187M17.1 & $\mathrm{RCC} 1$ & PRO2012 \\
\hline PTGER4 & PLS1 & RP11-368J21.1 & RPH3A & RN28S1 & PTPN20B \\
\hline RAD51AP1 & POLR2A & RPL12 & RPL23AP53 & RNF219 & PTPRD \\
\hline RAD9B & PQLC3 & RPL13 & RPS6KA1 & RP11-33B1.1 & PUS10 \\
\hline RCVRN & PRPH2 & RPL14 & RPUSD1 & RP11-440L14.1 & RABL3 \\
\hline RHOXF1 & PSG11 & RPL15 & RTL1 & RP11-571M6.6 & RALYL \\
\hline
\end{tabular}




\begin{tabular}{|c|c|c|c|c|c|}
\hline V1 (Polar angle) & V2 (Polar angle) & V3 (Polar angle) & V1 (polar angle) & V2 (polar angle) & V3 (polar angle) \\
\hline RIBC2 & PTGIS & RPL23 & RUSC1 & RPL23AP53 & RLN1 \\
\hline RNASE10 & RAB17 & RPL31 & SAPS1 & RPS6KA1 & RP1-286D6.2 \\
\hline RP11-195F19.1 & RAB7B & RPL31P11 & SCARF1 & RSL1D1 & RP11-33B1.1 \\
\hline RP11-45B20.3 & RAD21L1 & RPL36A & SCARNA17 & RWDD2B & RPN2 \\
\hline RP11-537P22.1 & RASL11B & RPL38 & SEPT6 & SAPS1 & RSC1A1 \\
\hline RP11-61008.1 & RP11-537P22.1 & RPL4 & SIPA1L2 & SDC1 & SERINC4 \\
\hline SAMD9 & RP11-89K11.1 & RPLP1 & SLC26A8 & SEMA6C & SF3B1 \\
\hline SCML4 & RP5-820A21.1 & RPN1 & SLC6A17 & SIGLEC8 & SGPP1 \\
\hline SCNN1A & RRN3P1 & RPP38 & SMARCC2 & SLC18A3 & SHANK2 \\
\hline SH3BP4 & SAMD9 & RPS12 & SPDYE2 & SLC32A1 & SLAMF9 \\
\hline SLC26A9 & SCFD2 & RPS17 & SPTBN4 & SLC4A9 & SLC19A1 \\
\hline SLC35F2 & SCNN1A & RPS19 & SR140 & SMCR7 & SLC25A51P1 \\
\hline SPANXN4 & SENP6 & RPS20 & SSX2B & SMPX & SLC26A2 \\
\hline SPATA19 & SERPINF2 & RPS21 & STK11 & SNCG & SLC2A3P1 \\
\hline SPINK4 & SEZ6L & RPS25 & STK19 & SNX24 & SLC36A4 \\
\hline SPRR2B & SGCA & RPS4Y2 & STK40 & SOAT2 & SLC38A4 \\
\hline STK31 & SIM2 & RPS6 & SYN3 & SSTR5 & SLFNL1 \\
\hline TDGF1 & SLC13A4 & RPS7 & SYP & ST14 & SNX30 \\
\hline TDO2 & SLC24A4 & RPSAP15 & TADA3L & STK40 & SPACA1 \\
\hline TDRD5 & SLC25A30 & RRN3P1 & TAF3 & TACC3 & SPAG4L \\
\hline TEKT5 & SLC35A3 & S100A11 & TAOK2 & TAF1 & SPDYE2 \\
\hline TK2 & SLC39A14 & S100P & TCEAL2 & TAF3 & SPRR1B \\
\hline TLR8 & SMR3A & SCNN1A & TFPI & TAS2R40 & TAS2R13 \\
\hline TMC5 & SOX17 & SDF4 & TNFAIP1 & TBX21 & TBX21 \\
\hline TMEM200B & SPATA4 & SERBP1 & TNFRSF25 & TCEAL1 & TFDP3 \\
\hline TMEM75 & ST8SIA6 & SH3TC1 & TNK2 & TCEAL2 & THEM4 \\
\hline TMPRSS4 & STK31 & SLC13A4 & TNRC6A & TDRKH & TMCO7 \\
\hline TNFAIP6 & SUSD2 & SLC19A3 & TSPY26P & TERT & TMEM190 \\
\hline TNFRSF14 & TAGLN & SLC2A1 & UBL4A & TGFA & TMEM66 \\
\hline TPH1 & TARP & SLC6A13 & UHRF1BP1 & THAP7 & TMPRSS3 \\
\hline TRAT1 & TEKT5 & SNRPF & UNC13B & TMEM195 & TNFSF15 \\
\hline TREML3 & TEX9 & SNX15 & USP28 & TMEM57 & TNR \\
\hline TRPC5OS & TFPI2 & SSR4P1 & VEGFA & TPBG & TPSAB1 \\
\hline TRPC6 & TM6SF2 & SUSD2 & VSIG8 & TRPM1 & TRAF3IP2 \\
\hline TTY8 & TMEM215 & TGFBR2 & VWC2 & TSPY26P & TSPY1 \\
\hline TUBB1 & TMEM79 & TIMP3 & WDR55 & UMPS & TSPY26P \\
\hline UPK1A & TNF & TMEM109 & WWOX & URI1 & TTC12 \\
\hline VCAM1 & TRIM51GP & TMEM150A & ZHX3 & WARS2 & UBE4A \\
\hline VIT & TTR & TMOD4 & ZMAT3 & WASL & UHRF1 \\
\hline VSX2 & ТTТY3В & TRAF7 & ZNF264 & wWOX & WISP1 \\
\hline
\end{tabular}


bioRxiv preprint doi: https://doi.org/10.1101/812560; this version posted October 21,2019 . The copyright holder for this preprint (which was not certified by peer review) is the author/funder, who has granted bioRxiv a license to display the preprint in perpetuity. It is made available under aCC-BY-NC-ND 4.0 International license.

\begin{tabular}{|c|c|c|c|c|c|}
\hline V1 (Polar angle) & V2 (Polar angle) & V/3 (Polar angle) & V1 (polar angle) & V2 (polar angle) & V3 (polar angle) \\
\hline WDR49 & UGT2B10 & TRAPPC2 & ZNF385D & ZBTB5 & XRCC2 \\
\hline XAGE1B & VSTM5 & TSPAN1 & ZNF485 & ZDHHC8 & XYLB \\
\hline XIRP1 & WNT2 & TTC7A & ZNF513 & ZFHX2 & ZBTB33 \\
\hline ZBTB3 & XIRP1 & U2AF1 & ZNF559 & ZNF213 & ZDHHC8 \\
\hline ZDHC15 & ZNF114 & ULK4 & ZNF585B & ZNF280B & ZNF300P1 \\
\hline ZFP92 & ZNF2 & WBSCR27 & ZNF641 & ZNF574 & ZNF449 \\
\hline ZNF705C & ZNF304 & WHAMML1 & ZNF660 & ZNF594 & ZNF585B \\
\hline ZNF732 & ZNF597 & XRRA1 & ZNF704 & ZNF704 & ZNF594 \\
\hline ZNF90 & ZNF799 & ZAR1 & ZNF749 & ZNF710 & ZNF772 \\
\hline ZWINT & ZP1 & ZGPAT & ZNF787 & ZNF771 & ZSCAN21 \\
\hline
\end{tabular}


bioRxiv preprint doi: https://doi.org/10.1101/812560: this version posted October 21. 2019. The copvriaht holder for this preprint (which was not certified by peer review) is the author/funder, who has granted bioRxiv a license to display the preprint in perpetuity. It is made available under aCC-BY-NC-ND 4.0 International license.

Supplementary

Table 2

\section{X chromosome genes correlated with eccentricity}

\begin{tabular}{|c|}
\hline CXorf5 \\
\hline DCAF8L2 \\
\hline EDA \\
\hline FLJ4635 \\
\hline H2AFB2 \\
\hline HDAC8 \\
\hline HS6ST2 \\
\hline IQSEC2 \\
\hline OR13H1 \\
\hline PABPC1L2A \\
\hline PGAM4 \\
\hline POF1B \\
\hline PRRG3 \\
\hline RAI2 \\
\hline SLITRK4 \\
\hline SMPX \\
\hline SNX12 \\
\hline SSX2B \\
\hline STS \\
\hline WMEM47 \\
\hline
\end{tabular}

